\title{
Manual de Laboratorio de Mecánica
}

ISBN (e) 978-958-8166-87-2

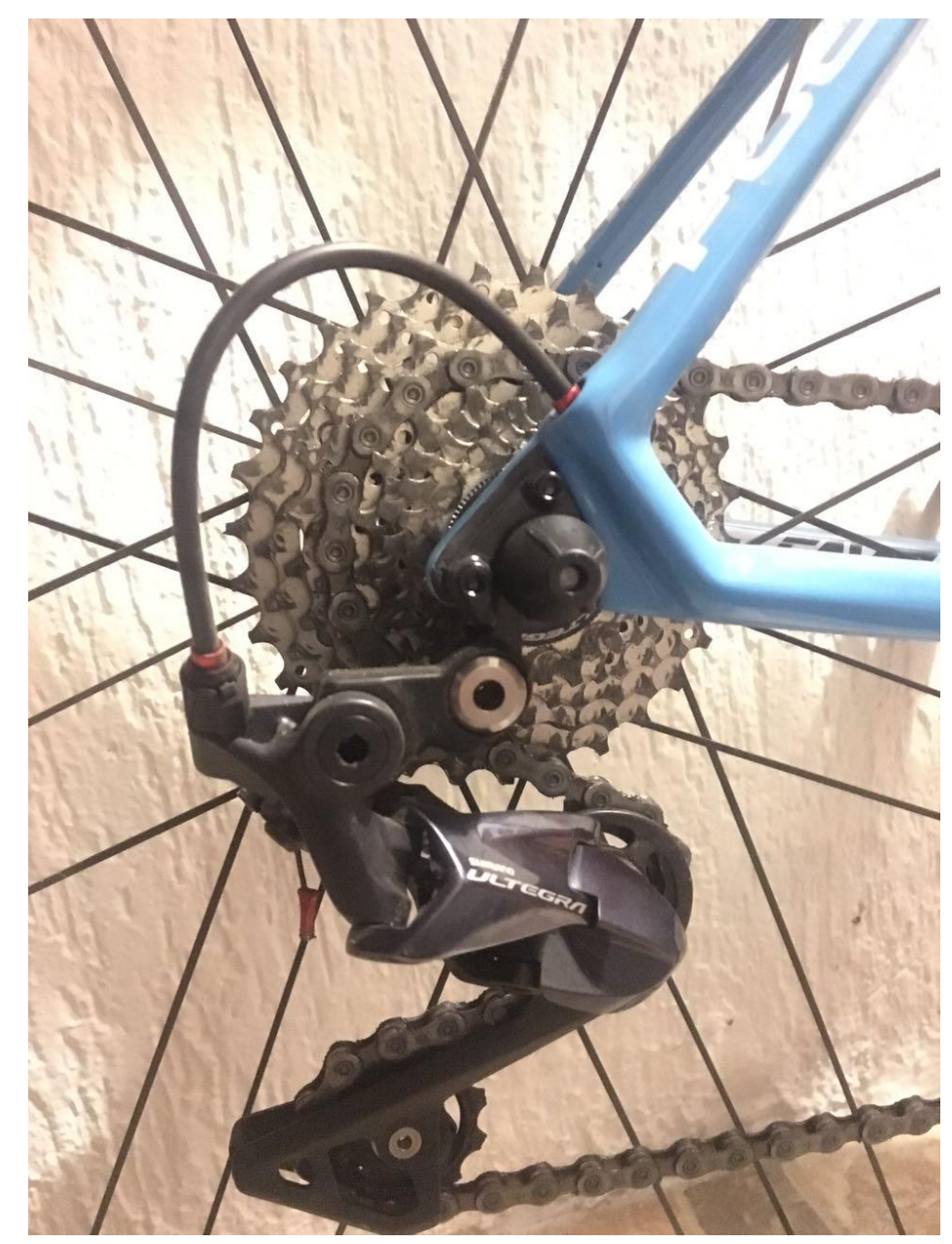

Ligia Beleño Montagut

Martha Lucía Barrera Pérez

Luis Alejandro Prada Martínez

\section{䑶}




\title{
UNIVERSIDAD AUTÓNOMA DE BUCARAMANGA
}

Manual de Laboratorio de Mecánica

ISBN (e) 978-958-8166-87-2

\author{
ALBERTO MONTOYA PUYANA \\ Rector \\ EULALIA GARCÍA BELTRÁN \\ Vicerrectora Académica \\ GILBERTO RAMÍREZ VALBUENA \\ Vicerrector administrativo y financiero \\ NÓHORA ISABEL NÁJERA ESTEBAN \\ Directora \\ Departamento de Matemáticas y Ciencias Naturales \\ LIGIA BELEÑO MONTAGUT \\ Departamento de Matemáticas y Ciencias Naturales \\ MARTHA LUCÍA BARRERA PÉREZ \\ Departamento de Matemáticas y Ciencias Naturales \\ LUIS ALEJANDRO PRADA MARTÍNEZ \\ Autores \\ JOSE OSCAR MACHADO ROMERO \\ Corrección de Estilo. \\ PUBLICACIONES UNAB \\ Producción \\ Universidad Autónoma de Bucaramanga \\ Avenida $42 \mathrm{~N}^{\circ} 48-11$ \\ Bucaramanga, Colombia \\ www.unab.edu.co
}

Las opiniones contenidas en esta obra no vinculan a la Institución, sino que son exclusiva responsabilidad de los autores, dentro de los principios democráticos de la cátedra libre y la libertad de expresión consagrados en el artículo $3^{\circ}$ del Estatuto General de la Corporación Universidad Autónoma de Bucaramanga. 
Tabla de Contenido

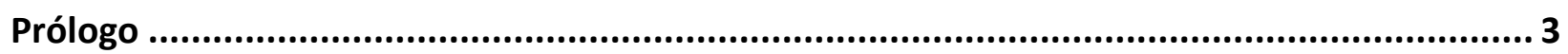

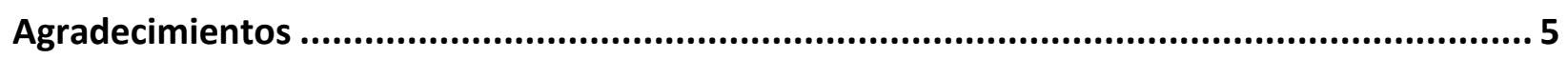

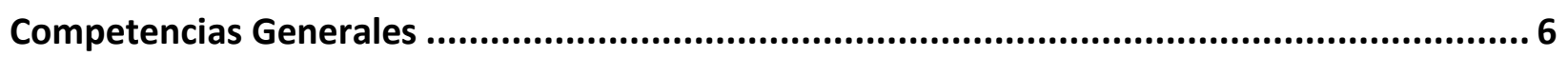

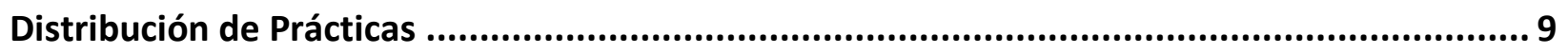

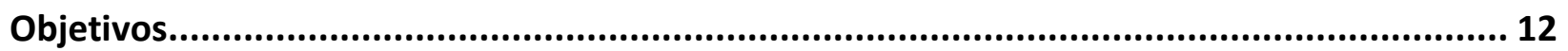

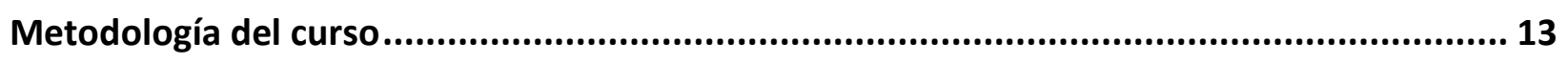

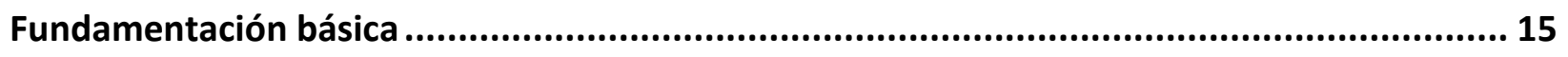

Sesión 1- Gráfica de Datos y Análisis de resultados ................................................. 23

Sesión 2- Medidas Fundamentales ........................................................................... 29

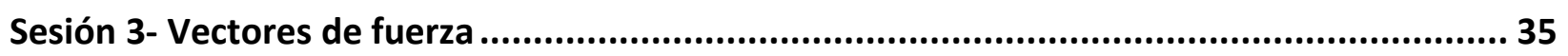

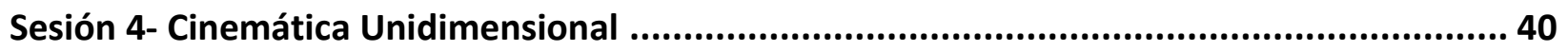

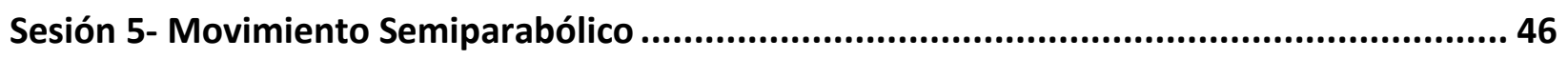

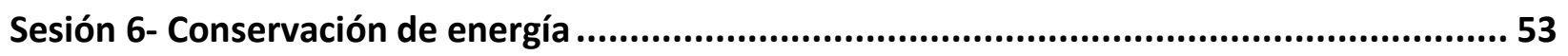

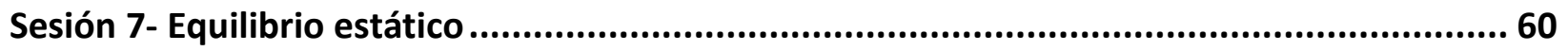




\section{Prólogo}

Este Manual de laboratorio es producto del análisis y trabajo continuo de los docentes de tiempo completo en la línea de física del departamento de Matemáticas y Ciencias Naturales. La finalidad del manual de laboratorio de Mecánica es apoyar tanto al alumno como a otros docentes que imparten la misma asignatura, de manera que reconozcan el entorno de trabajo y puedan preparar, previamente, la fundamentación requerida en cada una de las sesiones programadas.

Se resalta la importancia de la precisión en las medidas y en particular la elaboración y análisis gráfico, lo que para muchos alumnos es un punto de partida pues no están acostumbrados al uso de laboratorios en asignaturas anteriores, por lo tanto les permitirá, poco a poco, profundizar en la importancia del saber hacer en su formación como ingenieros.

Se espera que el estudiante prepare con anticipación la fundamentación teórica que se sugiere en cada guía apoyándose en los libros, simulaciones y trabajos similares que le permita crear una representación previa acerca del trabajo que realizará en el laboratorio. Los cálculos, evaluación de errores y conclusiones serán el resultado del análisis de tablas y gráficas realizadas durante el experimento.

La primera práctica se enfoca en la realización e interpretación de gráficas y análisis para un problema particular. En las prácticas sucesivas se han diseñado sesiones paralelas a la teoría, de acuerdo a los temas: Medidas fundamentales enfocado hacia las medidas de las cantidades físicas fundamentales y sus aplicaciones, vectores analizando sistemas en equilibrio, cinemática en una y dos dimensiones, conservación de energía y su análisis en diferentes puntos de una trayectoria, estática y las características de un cuerpo rígido en las cuales aplicará los conceptos aprendidos en el curso teórico y proyectará su conocimiento hacia aplicaciones en la ingeniería. 
Esperamos que el uso de este material por parte de los estudiantes les permita desarrollar sus habilidades y ampliar su visión como futuros ingenieros. Adicionalmente, las ideas y sugerencias resultado de la aplicación de este manual en las clases serán bien recibidas.

\section{Los autores}




\section{Agradecimientos}

Los autores agradecen a la Universidad Autónoma de Bucaramanga por el espacio académico de laboratorio el cual fortalece las habilidades de nuestros alumnos de ingeniería y a su vez representa un gran apoyo a los docentes en sus labores académicas.

Por otra parte se agradece a los compañeros y a los alumnos que con sus ideas y observaciones han participado de manera activa en este constante proceso de construcción.

De manera especial se valora el apoyo del Ingeniero Julián Andrés Martínez por su constante apoyo como auxiliar del laboratorio. 


\section{Competencias Generales}

\section{Respecto al trabajo en equipo}

- Propender por el logro de los objetivos del equipo de trabajo con el fin de cumplir con los compromisos que requiere el desarrollo de la práctica experimental.

- Valorar las ideas y opiniones de los demás compañeros del equipo para construir el análisis del experimento, a partir de las mismas.

- Colaborar activamente con sus compañeros del equipo, con la propuesta de soluciones.

- Facilitar la comunicación, manteniendo relaciones productivas y respetuosas con los demás compañeros.

\section{Respecto a la iniciativa}

- Participar activamente aportando ideas y estimulando a sus compañeros de grupo a trabajar de forma conjunta y proactiva.

- Tomar decisiones inmediatas cuando se detecte algún error en el procedimiento realizado, con el fin de reducir el riesgo de obtener resultados inconsistentes en el análisis del experimento.

- Analizar con profundidad las situaciones propuestas en las guías de laboratorio.

\section{Respecto a la flexibilidad}

- Adaptarse a cada situación que se presente durante el desarrollo de la experiencia de laboratorio.

- Propender por la tolerancia hacia los diversos puntos de vista de sus compañeros, con el fin de mantener la armonía y favorecer la toma de las mejores decisiones del grupo y así alcanzar las metas propuestas. 


\section{Respecto a la comunicación}

- Facilitar la comunicación con los demás miembros del grupo, socializando los resultados obtenidos durante la práctica.

- Escuchar activamente a sus compañeros, aportando la retroalimentación necesaria y analizando las participaciones de los demás para el logro de los objetivos de la experiencia realizada.

\section{Respecto a la responsabilidad}

- Comprometerse con todas las actividades previas a la realización del experimento, así como durante el desarrollo del mismo.

- Estructurar el tiempo del desarrollo de la práctica con el fin de poder entregar los resultados del informe dentro del plazo definido en el reglamento del laboratorio de Física.

- Asumir con responsabilidad los errores cometidos por su equipo de trabajo, ya sea en la toma de mediciones, en el manejo de equipos o en la realización del informe, estableciendo con prontitud los correctivos necesarios para enmendarlos.

\section{Respecto al conocimiento}

- Expresar e interpretar conceptos y opiniones de forma oral y escrita, de acuerdo al contexto, con un buen manejo del vocabulario y aplicando correctamente las normas gramaticales del lenguaje. Análisis de un modelo específico al cual dará explicación con base en la toma de datos y análisis gráfico y de resultados.

- Desarrollar y aplicar el razonamiento matemático, para la formulación de modelos que permitan explicar los fenómenos naturales estudiados en cada una de las prácticas de laboratorio. 
- Utilizar las herramientas tecnológicas para el tratamiento y el uso de la información obtenida en la toma de mediciones, con el fin de producir y presentar el análisis de los resultados en el reporte final de cada laboratorio. 
Distribución de Prácticas

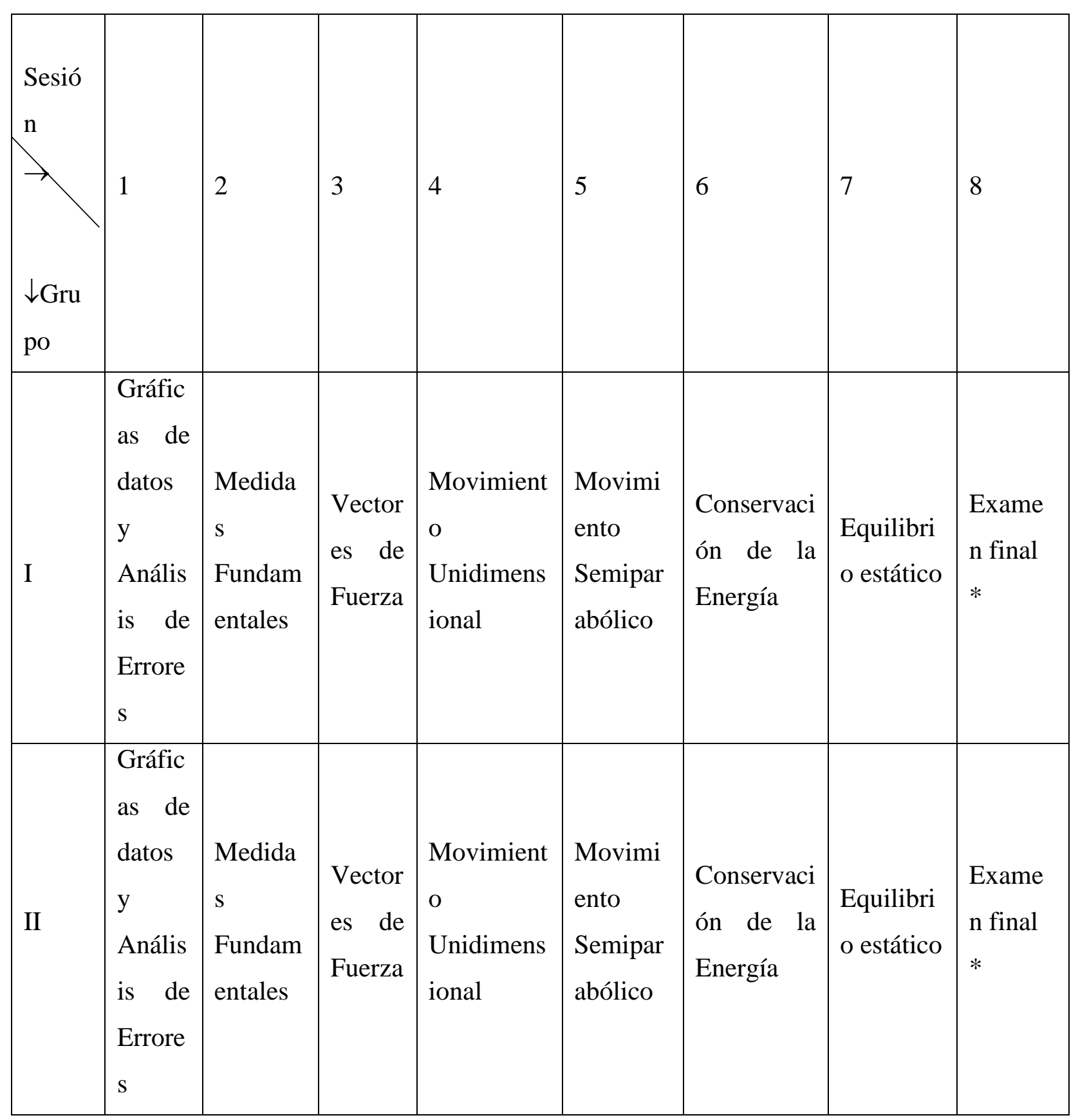




\begin{tabular}{|c|c|c|c|c|c|c|c|c|}
\hline III & $\begin{array}{l}\text { Gráfic } \\
\text { as de } \\
\text { datos } \\
\text { y } \\
\text { Anális } \\
\text { is de } \\
\text { Errore } \\
\text { s }\end{array}$ & $\begin{array}{l}\text { Medida } \\
\text { s } \\
\text { Fundam } \\
\text { entales }\end{array}$ & $\begin{array}{l}\text { Vector } \\
\text { es de } \\
\text { Fuerza }\end{array}$ & $\begin{array}{l}\text { Movimient } \\
\text { o } \\
\text { Unidimens } \\
\text { ional }\end{array}$ & $\begin{array}{l}\text { Movimi } \\
\text { ento } \\
\text { Semipar } \\
\text { abólico }\end{array}$ & $\begin{array}{l}\text { Conservaci } \\
\text { ón de la } \\
\text { Energía }\end{array}$ & $\begin{array}{l}\text { Equilibri } \\
\text { o estático }\end{array}$ & $\begin{array}{l}\text { Exame } \\
\text { n final } \\
*\end{array}$ \\
\hline IV & $\begin{array}{l}\text { Gráfic } \\
\text { as de } \\
\text { datos } \\
\text { y } \\
\text { Anális } \\
\text { is de } \\
\text { Errore } \\
\mathrm{s}\end{array}$ & $\begin{array}{l}\text { Medida } \\
\text { s } \\
\text { Fundam } \\
\text { entales }\end{array}$ & $\begin{array}{l}\text { Vector } \\
\text { es de } \\
\text { Fuerza }\end{array}$ & $\begin{array}{l}\text { Movimient } \\
\text { o } \\
\text { Unidimens } \\
\text { ional }\end{array}$ & $\begin{array}{l}\text { Movimi } \\
\text { ento } \\
\text { Semipar } \\
\text { abólico }\end{array}$ & $\begin{array}{l}\text { Conservaci } \\
\text { ón de la } \\
\text { Energía }\end{array}$ & $\begin{array}{l}\text { Equilibri } \\
\text { o estático }\end{array}$ & $\begin{array}{l}\text { Exame } \\
\text { n final } \\
*\end{array}$ \\
\hline V & $\begin{array}{l}\text { Gráfic } \\
\text { as de } \\
\text { datos } \\
\text { y } \\
\text { Anális } \\
\text { is de } \\
\text { Errore } \\
\mathrm{s}\end{array}$ & $\begin{array}{l}\text { Medida } \\
\mathrm{s} \\
\text { Fundam } \\
\text { entales }\end{array}$ & $\begin{array}{l}\text { Vector } \\
\text { es de } \\
\text { Fuerza }\end{array}$ & $\begin{array}{l}\text { Movimient } \\
\text { o } \\
\text { Unidimens } \\
\text { ional }\end{array}$ & $\begin{array}{l}\text { Movimi } \\
\text { ento } \\
\text { Semipar } \\
\text { abólico }\end{array}$ & $\begin{array}{l}\text { Conservaci } \\
\text { ón de la } \\
\text { Energía }\end{array}$ & $\begin{array}{l}\text { Equilibri } \\
\text { o estático }\end{array}$ & $\begin{array}{l}\text { Exame } \\
\text { n final } \\
*\end{array}$ \\
\hline VI & $\begin{array}{l}\text { Gráfic } \\
\text { as de } \\
\text { datos } \\
\text { y } \\
\text { Anális } \\
\text { is de }\end{array}$ & $\begin{array}{l}\text { Medida } \\
\mathrm{s} \\
\text { Fundam } \\
\text { entales }\end{array}$ & $\begin{array}{l}\text { Vector } \\
\text { es de } \\
\text { Fuerza }\end{array}$ & $\begin{array}{l}\text { Movimient } \\
\text { o } \\
\text { Unidimens } \\
\text { ional }\end{array}$ & $\begin{array}{l}\text { Movimi } \\
\text { ento } \\
\text { Semipar } \\
\text { abólico }\end{array}$ & $\begin{array}{l}\text { Conservaci } \\
\text { ón de la } \\
\text { Energía }\end{array}$ & $\begin{array}{l}\text { Equilibri } \\
\text { o estático }\end{array}$ & $\begin{array}{l}\text { Exame } \\
\text { n final } \\
*\end{array}$ \\
\hline
\end{tabular}




\begin{tabular}{|c|c|c|c|c|c|c|c|c|}
\hline & $\begin{array}{l}\text { Errore } \\
\mathrm{S}\end{array}$ & & & & & & & \\
\hline VII & $\begin{array}{l}\text { Gráfic } \\
\text { as de } \\
\text { datos } \\
\text { y } \\
\text { Anális } \\
\text { is de } \\
\text { Errore } \\
\mathrm{s}\end{array}$ & $\begin{array}{l}\text { Medida } \\
\text { s } \\
\text { Fundam } \\
\text { entales }\end{array}$ & $\begin{array}{l}\text { Vector } \\
\text { es de } \\
\text { Fuerza }\end{array}$ & $\begin{array}{l}\text { Movimient } \\
\text { o } \\
\text { Unidimens } \\
\text { ional }\end{array}$ & $\begin{array}{l}\text { Movimi } \\
\text { ento } \\
\text { Semipar } \\
\text { abólico }\end{array}$ & $\begin{array}{l}\text { Conservaci } \\
\text { ón de la } \\
\text { Energía }\end{array}$ & $\begin{array}{l}\text { Equilibri } \\
\text { o estático }\end{array}$ & $\begin{array}{l}\text { Exame } \\
\text { n final } \\
*\end{array}$ \\
\hline VIII & $\begin{array}{l}\text { Gráfic } \\
\text { as de } \\
\text { datos } \\
\text { y } \\
\text { Anális } \\
\text { is de } \\
\text { Errore } \\
\text { s }\end{array}$ & $\begin{array}{l}\text { Medida } \\
\text { s } \\
\text { Fundam } \\
\text { entales }\end{array}$ & $\begin{array}{l}\text { Vector } \\
\text { es de } \\
\text { Fuerza }\end{array}$ & $\begin{array}{l}\text { Movimient } \\
\text { o } \\
\text { Unidimens } \\
\text { ional }\end{array}$ & $\begin{array}{l}\text { Movimi } \\
\text { ento } \\
\text { Semipar } \\
\text { abólico }\end{array}$ & $\begin{array}{l}\text { Conservaci } \\
\text { ón de la } \\
\text { Energía }\end{array}$ & $\begin{array}{l}\text { Equilibri } \\
\text { o estático }\end{array}$ & $\begin{array}{l}\text { Exame } \\
\text { n final } \\
*\end{array}$ \\
\hline
\end{tabular}




\section{Objetivos}

Fortalecer en el estudiante de ingeniería de la UNAB la capacidad crítica, reflexiva y analítica que fortalezca el pensamiento científico y tecnológico, como competencia propia de la investigación.

Incorporar pedagogías activas que favorezcan el aprendizaje significativo y mejorar los ambientes de enseñanza.

Conducir al estudiante hacia el descubrimiento de la ciencia mediante el uso del método científico. 


\section{Metodología del curso}

Para el buen desempeño durante las prácticas del laboratorio de Física se deben observar las siguientes recomendaciones:

- Durante la práctica, evite mantener dispositivos tales como: teléfonos celulares y demás objetos personales sobre el mesón de trabajo, estos pueden eventualmente convertirse en elementos que desvían su atención y la de sus compañeros. En su mesón de trabajo solamente debe permanecer su computador y sus herramientas de trabajo. Para ubicar su material que no hace parte de la práctica, el laboratorio dispone del gabinete inferior derecho del mesón de trabajo correspondiente al grupo. Se recomienda no perturbar el trabajo de los compañeros, evite visitar los mesones vecinos y las visitas de personas ajenas a la sesión del laboratorio.

- El laboratorio requiere de la lectura previa de los temas que se relacionan con la práctica correspondiente según indicaciones presentadas en este manual, como preparación para el quiz de entrada que se realizará en cada sesión.

- Para la toma de datos, los subgrupos se conformarán el primer día de clase y se asignará un número para identificar las prácticas que realizarán durante el semestre sin confusión alguna. La duración de cada experiencia es de 4 horas según las fechas estipuladas en el anexo 2 y consta de aproximadamente 2 horas para la toma de datos y 2 horas para la elaboración del informe.

- Al inicio de cada sesión el auxiliar de laboratorio le hará entrega formal de los materiales de trabajo a cada grupo y recogerá un documento de identificación por mesa de trabajo. Al finalizar la práctica los estudiantes deberán hacer la entrega al auxiliar de los elementos suministrados y él a su vez les devolverá el documento. 
- La práctica de laboratorio no tiene derecho a supletorio. En casos excepcionales se debe presentar una excusa, por escrito, dirigida al docente de laboratorio, quien tramitará dicha solicitud ante el equipo docente, para determinar la aceptación o no de dicha excusa. En caso de ser rechazada el estudiante tendrá nota de 0.0 (cero punto cero) en la sesión correspondiente y en caso de ser aceptada, se recuperará la experiencia en una única sesión programada por el equipo docente.

- El laboratorio aporta el $20 \%$ de la nota de la asignatura de Física: $10 \%$ para el primer corte (donde las prácticas de laboratorio serán el 7\% y los Quices 3\%); el 10\% restante (correspondiente a las demás prácticas serán el 3\%, y los Quices 2\% además de un examen final que aporta un 5\%) para el segundo corte, según las fechas estipuladas por la Universidad. 
Fundamentación básica

1. Sistemas Internacionales de Unidades

1.1. Magnitudes Fundamentales: Se definen por sí mismas, son patrones de referencia.

\begin{tabular}{|l|l|}
\hline Magnitud & Unidad \\
\hline Longitud (L) & Metro (m) \\
\hline Masa (M) & Kilogramo (kg) \\
\hline Tiempo (t) & Segundo (s) \\
\hline Temperatura & Kelvin (k) \\
\hline Intensidad de corriente & Amperio (A) \\
\hline Intensidad luminosa & Candela (cd) \\
\hline Cantidad de sustancia & Número de moles (mol) \\
\hline
\end{tabular}

1.2. Magnitudes derivadas: Están definidas en función de las fundamentales.

\begin{tabular}{|l|l|}
\hline Magnitud & Unidad \\
\hline Área o superficie (S) & $\mathrm{m}^{2}$ \\
\hline Volumen (V) & $\mathrm{m}^{3}$ \\
\hline Densidad (d) & $\mathrm{Kg} / \mathrm{m}^{3}$ \\
\hline Velocidad (v) & $\mathrm{m} / \mathrm{s}$ \\
\hline Aceleración & $\mathrm{m} / \mathrm{s}^{2}$ \\
\hline Fuerza (F) & $\mathrm{N}($ Newton $)=\mathrm{kg} * \mathrm{~m} / \mathrm{s}^{2}$ \\
\hline Presión (P) & $\mathrm{Pa}\left(\right.$ Pascal) $=\mathrm{N} / \mathrm{m}^{2}$ \\
\hline Energía (E) & $\mathrm{J}($ Julio $)=\mathrm{N} * \mathrm{~m}$ \\
\hline
\end{tabular}




\section{Prefijos del Sistema Internacional (SI)}

Los prefijos que indican varias potencias de 10 se usan con estas tres unidades básicas.

\begin{tabular}{|l|l|l|l|l|l|}
\hline Factor & Prefijo & Símbolo & Factor & Prefijo & Símbolo \\
\hline $10^{18}$ & Exa & E & $10^{-1}$ & deci & d \\
\hline $10^{15}$ & Peta & P & $10^{-2}$ & centi & c \\
\hline $10^{12}$ & Tera & T & $10^{-3}$ & mili & $\mathrm{m}$ \\
\hline $10^{9}$ & Giga & G & $10^{-6}$ & micro & $\mu$ \\
\hline $10^{6}$ & Mega & M & $10^{-9}$ & nano & $\mathrm{n}$ \\
\hline $10^{3}$ & Kilo & K & $10^{-12}$ & pico & $\mathrm{p}$ \\
\hline $10^{2}$ & Hecto & h & $10^{-15}$ & femto & f \\
\hline $10^{1}$ & deca & de & $10^{-18}$ & atto & a \\
\hline
\end{tabular}

\section{Tablas de conversión de unidades}

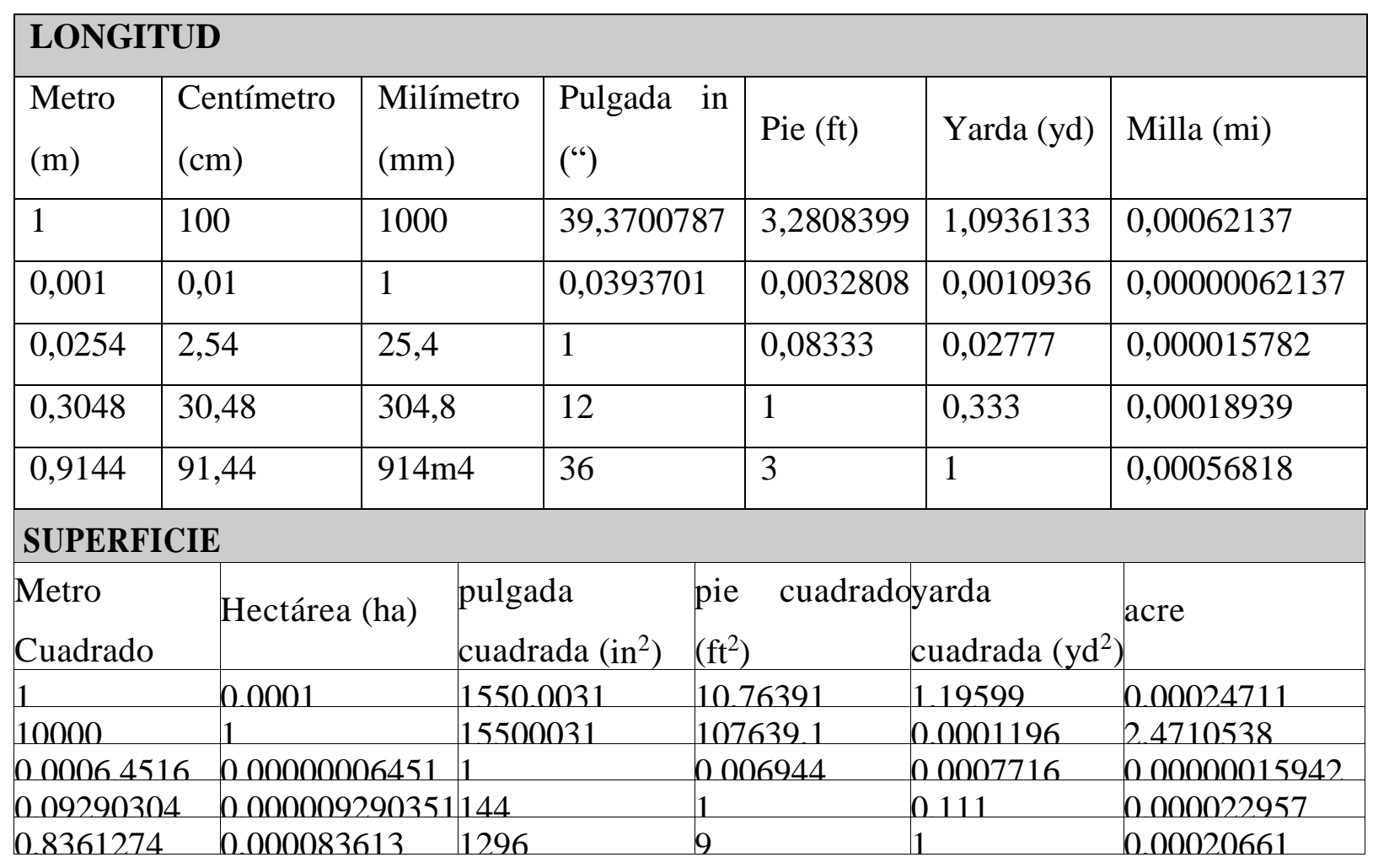




\begin{tabular}{|c|c|c|c|c|c|}
\hline 4046.856 & 0.4046856 & 6272640 & 43560 & 4840 & 1 \\
\hline \multicolumn{6}{|c|}{ VOLUMEN } \\
\hline \multicolumn{2}{|c|}{ metro cúbicoLitro } & \multicolumn{2}{|c|}{ pie cúbico $\mathrm{ft}^{3}$ galón } & galón imper & abarril de petróleo \\
\hline 1 & 1000 & 35.3146667 & 264.17205 & 219.96923 & 6.2898108 \\
\hline 0.001 & 1 & 0.0353147 & 0.2641721 & 0.2199692 & 0.0062898 \\
\hline 0.0283168 & 28.3168466 & 1 & 7.4805195 & 6.2288349 & 0.1781076 \\
\hline 0.0037854 & 3.7854118 & 0.1336806 & 1 & 0.8326741 & 0.0238095 \\
\hline 0.0045461 & 4.5460904 & 0.1635437 & 1.20095 & 1 & 0.028594 \\
\hline 1589873 & 158987295 & 56145833 & $42^{\prime}$ & 34.9723128 & 1 \\
\hline \multicolumn{2}{|c|}{$1 \mathrm{gal}(\mathrm{USA})=3,78541 \mathrm{dm}^{3}$} & $1 \mathrm{ft}^{3}=0$ & $83 \mathrm{~m}^{3}$ & & \\
\hline
\end{tabular}

\begin{tabular}{|c|c|c|c|c|c|}
\hline \multicolumn{6}{|c|}{ UNIDA DE PRESIÓN } \\
\hline $\begin{array}{l}\text { Kilopascal } \mathrm{kN} \\
/ \mathrm{m}^{2}\end{array}$ & $\begin{array}{l}\text { Atmósfera } \\
\text { técnica } \\
\mathrm{Kgf} / \mathrm{cm}^{2}\end{array}$ & $\begin{array}{l}\text { Milímetro } \\
\text { de c.Hg } \\
\left(0^{\circ} \mathrm{C}\right)\end{array}$ & $\begin{array}{l}\text { Metros de } \\
\text { c.agua } \\
\left(4^{\circ} \mathrm{C}\right)\end{array}$ & $\begin{array}{l}\text { libras por } \\
\text { pulgada } \\
\text { lib/in } 2\end{array}$ & $\begin{array}{l}\mathrm{Bar} \\
100000 \\
\mathrm{~Pa}\end{array}$ \\
\hline 1 & 0,0101972 & 7,5006278 & 0,1019745 & 0,1450377 & 0,01 \\
\hline 98,0665 & 1 & 735,560217 & 1000028 & 14,2233433 & 0,980665 \\
\hline 0,1333222 & 0,0013595 & 1 & 0,0135955 & 193367 & 0,0013332 \\
\hline 9,8063754 & 0,0999972 & 73,5539622 & 1 & 1,4222945 & 0,0980638 \\
\hline 6,8947573 & 0,070307 & 51,7150013 & 0,7030893 & 1 & 0,0689476 \\
\hline 100 & 1,0197162 & 750,062679 & 10,1974477 & 14,5037738 & 1 \\
\hline \multicolumn{6}{|c|}{$\begin{array}{l}1 \text { in } \mathrm{H} 2 \mathrm{O}\left(60^{\circ} \mathrm{F}=15,55^{\circ} \mathrm{C}\right)=0,248843 \mathrm{kP} \quad \text { in } \mathrm{H} 2 \mathrm{O}\left(60^{\circ} \mathrm{F}=20^{\circ} \mathrm{C}\right)=0,248641 \mathrm{kPa} \\
1 \text { atmósfera física }(\text { Atm })=101,325 \mathrm{kPa}=760 \mathrm{~mm} \mathrm{Hg}\end{array}$} \\
\hline
\end{tabular}

\begin{tabular}{|c|c|c|c|c|c|}
\hline \multicolumn{6}{|c|}{ Energía (Calor y Trabajo) } \\
\hline $\begin{array}{l}\text { Kilojulio } \\
\text { kJ }\end{array}$ & $\begin{array}{l}\mathrm{kW} / \mathrm{hora} \\
\mathrm{kW} h\end{array}$ & $\begin{array}{l}\text { Hourse } \\
\text { power/hor } \\
\text { a USA } \\
550 \\
\text { ft.lbf/seg }\end{array}$ & $\begin{array}{l}\text { Caballo/h } \\
\text { ora } 75 \\
\text { m.Kgf/seg } \\
\text { CV.h }\end{array}$ & $\begin{array}{l}\text { Kilocalorí } \\
\text { a (IT) } \\
\text { Kcal(IT) } \\
\text { Kcal (IT) }\end{array}$ & $\begin{array}{l}\text { British } \\
\text { Therm } \\
\text { al Unit } \\
\text { Btu } \\
\text { (IT) }\end{array}$ \\
\hline
\end{tabular}




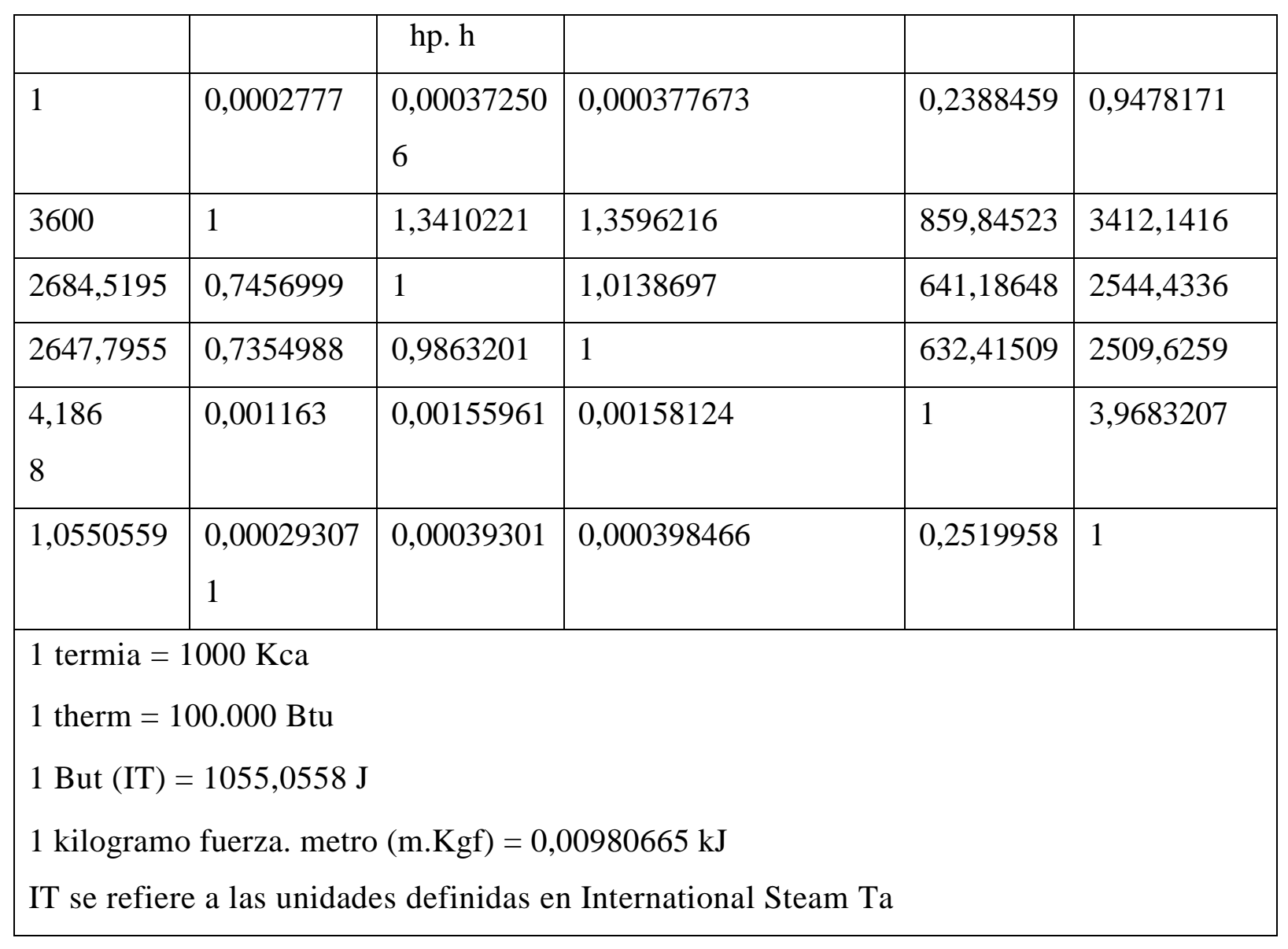

\begin{tabular}{|l|l|l|l|l|l|}
\hline \multicolumn{2}{|l|}{ Macrounidades Energéticas } \\
\hline $\begin{array}{l}\text { Terajulio } \\
\text { TJ }\end{array}$ & $\begin{array}{l}\text { Gigavatio } \\
\text { hora GW } \\
\text { h }\end{array}$ & $\begin{array}{l}\text { Teracaloría } \\
(\mathrm{IT}) \text { Tcal } \\
(\mathrm{IT})\end{array}$ & $\begin{array}{l}\text { Ton. } \\
\text { Equivalente } \\
\text { de carbón } \\
\text { Tec }\end{array}$ & $\begin{array}{l}\text { Ton. } \\
\text { Equivalente } \\
\text { de petróleo } \\
\text { Tep }\end{array}$ & $\begin{array}{l}\text { Barril de } \\
\text { petróleo } \\
\text { día-año bd }\end{array}$ \\
\hline 1 & 0,2727 & 0,2388459 & 34,1208424 & 23,8845897 & 0,4955309 \\
\hline 3,6 & 1 & 0,8598452 & 122,8350326 & 85,9845228 & 1,7839113 \\
\hline 4,1868 & 1,163 & 1 & 142,8571429 & 100 & 2,0746888 \\
\hline 0,0293076 & 0,008141 & 0,007 & 1 & 0,7 & 0,0145228 \\
\hline 0,041868 & 0,01163 & 0,01 & 1,4285714 & 1 & 0,0207469 \\
\hline 2,0180376 & 0,560568 & 0,482 & 68,8571429 & 48,2 & 1 \\
\hline
\end{tabular}




\begin{tabular}{|l|l|l|l|l|l|}
\hline \multicolumn{2}{|l|}{ Potencia } \\
\hline $\begin{array}{l}\text { Kilovatio } \\
\mathrm{kW}\end{array}$ & $\begin{array}{l}\text { Kilocaloría/hora } \\
\text { Kcal (IT)/h }\end{array}$ & $\begin{array}{l}\text { Btu } \\
(\mathrm{IT}) / \mathrm{hora} \\
\text { Btu (IT)/h }\end{array}$ & $\begin{array}{l}\text { Horse power } \\
\text { (USA) hp }\end{array}$ & $\begin{array}{l}\text { Caballo } \\
\text { vapor } \\
\text { métrico CV }\end{array}$ & $\begin{array}{l}\text { Tonelada de } \\
\text { refrigeración }\end{array}$ \\
\hline 1 & 859,84523 & 3412,1416 & 1,3410221 & 1,3596216 & 0,2843494 \\
\hline 0,001163 & 1 & 3,9683207 & 0,0015596 & 0,0015812 & 0,0003307 \\
\hline 0,00029307 & 0,2519958 & 1 & 0,00039301 & 0,00039847 & 0,000083335 \\
\hline 0,7456999 & 641,18648 & 2544,4336 & 1 & 1,0138697 & 0,2120393 \\
\hline 0,7354988 & 632,41509 & 2509,6259 & 0,9863201 & 1 & 0,2091386 \\
\hline 3,5168 & 3023,9037 & 11999,82 & 4,7161065 & 4,7815173 & 1 \\
\hline 1 caballo vapor (métrico $>75 \mathrm{~m} \mathrm{kgf/seg}=735,499 \mathrm{~W}$ & & \\
\hline 1 Horse power (USA) mecánico $=550 \mathrm{ft}$ I bf/seg & & & \\
\hline
\end{tabular}

\section{Temperatura}

Temperatura en ${ }^{\circ} \mathrm{C}=\left({ }^{\circ} \mathrm{F}-32\right) / 1,8$

Temperatura en ${ }^{\circ} \mathrm{F}=1,8 *{ }^{\circ} \mathrm{C}+32$

Temperatura ${ }^{\circ} \mathrm{K}={ }^{\circ} \mathrm{C}+273,14$

\section{Análisis dimensional}

El método del análisis dimensional es muy potente para resolver problemas físicos. Las dimensiones se pueden tratar como cantidades algebraicas.

\section{Notación científica}

Expresar un número con un solo dígito entero y una parte decimal seguida de una potencia de diez.

Ejemplo: 
$1210=1,21 \times 10^{3}$

$0,00456=4,56 \times 10^{-3}$

\section{Conceptos básicos}

6.1. Precisión: Es la variación de magnitud más pequeña que puede apreciar el aparato con resultados iguales al repetir varias veces la medida.

6.2. Exactitud: Mide la concordancia entre el valor hallado y el valor real de la medida; cuanto más cercano esté al valor real, más exacta será la medida.

\section{Cifras significativas}

Es el número de dígitos conocidos con certeza en una medida. Cuando calcule un resultado a partir de varios números medidos, cada uno de ellos con cierta precisión, deberá expresar el resultado con el número correcto de cifras significativas.

7.1. Son significativas: Todas las cifras distintas de cero. Ejemplo: $359 \mathrm{~cm}$ tiene tres cifras. Los ceros después de una cifra entera. Ejemplo: 3,05 tiene tres cifras, 3,00 tiene tres cifras

7.2. No son significativas: Los ceros antes de la coma decimal y los ceros inmediatamente después de la coma hasta el primer número entero. Ejemplos: 0,0067 tiene dos cifras significativas; 0,345 tiene tres cifras significativas; 0,000300 tiene tres cifras significativas.

7.3. Suma de números. El Resultado se da con las cifras significativas del número menos preciso: $2,345+0,11=2,356=2,4$ con dos cifras por redondeo.

7.4. Multiplicación y división. El resultado se da con el valor más pequeño de cifras:

$2,25 \times 2,1=4,725=4,7$

$2,45 / 2,2=1,113=1,1$

7.5. Redondeo. Si el número es mayor o igual a cinco (5) se aumenta en una unidad la anterior. Si el número es menor de cinco (5) no se aumenta y se eliminan las restantes cifras. 


\section{Ejemplos:}

Hasta la décima: $1,29=1,3 ; 1,24=1,2$

Hasta la centésima: $6,458=4,46 ; 9,342=9,34$

\section{Tipos de Errores.}

- Error sistemático: Aparece repentinamente debido al error del aparato o impericia del experimentador.

- Error Accidental: Error por azar, el experimentador comete puntualmente un fallo; con muchas medidas se elimina.

8.1. Imprecisión de una medida. Llamado error absoluto o diferencia entre el valor medido y el real.

Para un aparato de medida coincide con su precisión. En una regla que aprecia milímetros, el error absoluto sería de $1 \mathrm{~mm}$.

La medida se expresa: $\mathrm{x}=$ valor medio $(+/-)$ cota de error.

Un cronómetro (aprecia 0,01 segundos), t = 2 (+/-) 0,01 el valor real estará entre 1,99 y 2,01.

Para varias medidas se hace la media aritmética de valores medidos y de errores absolutos.

Ejemplos:

Tiempos $=1,25 \mathrm{~s} ; 1,26 \mathrm{~s} ; 1,24 \mathrm{~s} ; 1,23 \mathrm{~s}$

Valor medio $=(1,25+1,26+1,24+1,23) / 4=1,245=1,24 \mathrm{~s}$

8.2. Error Relativo. Es el cociente entre el error absoluto y el valor verdadero.

Notación: Error Relativo $(\mathrm{Er}) \quad \mathrm{Er}=(\mathrm{Ea} / \mathrm{x}) 100 \%$ 
A menor error relativo más precisa será la medida y viceversa.

\section{La Densidad}

Es una magnitud derivada definida como el cociente entre la masa del cuerpo y su volumen. Se mide en el S.I. en $\mathrm{Kg} / \mathrm{m}^{3}$.

La masa se mide en un balanza y el volumen se calcula por geometría si es regular o bien por desplazamiento de agua en una probeta si es irregular. 


\section{Sesión 1- Gráfica de Datos y Análisis de resultados}

\section{Competencias}

Al finalizar la práctica el estudiante estará en capacidad de:

- Encontrar leyes empíricas a partir de datos provenientes de experimentos realizados que permitan describir el comportamiento físico entre las variables involucradas en la medición.

- Comparar ideas y opiniones para la toma de decisiones y planes respecto a la temática estudiada junto con sus compañeros de grupo.

\section{Procedimiento}

En esta actividad se desea explorar la relación entre el tamaño de las hojas de una misma planta, representado a través de su longitud con respecto a su masa. Para este ejercicio se cuenta con los datos experimentales medidos para un conjunto de hojas de una variedad de planta llamada Adelfa o Nerium, conocida comúnmente como laurel (Ver la figura 1). Los datos se muestran en la

\section{Tabla 1.}

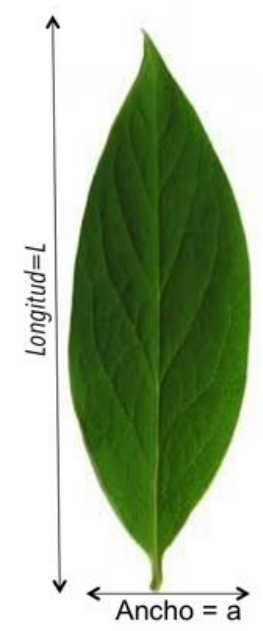


Figura 1. Hoja de Adelfa o Nerium, conocida comúnmente como laurel. Imagen tomada de http://www.lesarbres.fr/magnolia.html

Tabla 1. Longitud, ancho y masa de distintas hojas de laurel. Tomada de: Experimentos de Física usando las TIC y elementos de bajo costo. Autor: Salvador Gil. Editorial Omega. 2014. 


\begin{tabular}{|l|l|l|}
\hline Longitud $(\mathrm{cm})$ & Ancho $(\mathrm{cm})$ & Masa $(\mathrm{g})$ \\
\hline 8,5 & 11,0 & 0,278 \\
\hline 9,4 & 13 & 0,395 \\
\hline 10,0 & 14,0 & 0,435 \\
\hline 10,4 & 15,0 & 0,454 \\
\hline 11,6 & 16,0 & 0,602 \\
\hline 12,1 & 17,0 & 0,656 \\
\hline 12,3 & 17,0 & 0,671 \\
\hline 14,0 & 18,5 & 0,718 \\
\hline 14,4 & 23,0 & 1,033 \\
\hline 16,0 & 26,0 & 1,478 \\
\hline 16,5 & 24,0 & 1,263 \\
\hline 17,3 & 26,0 & 1,308 \\
\hline 20,5 & 31,0 & 2,045 \\
\hline 20,3 & 30,0 & 1,946 \\
\hline 22,2 & 36,0 & 2,424 \\
\hline 25,0 & 41,0 & 3,246 \\
\hline
\end{tabular}

\section{Análisis de datos y resultados}

Nota: La mayoría de hojas de cálculo, como Excel de Microsoft, disponen de herramientas de ajuste de curvas, es decir tienen la capacidad de estimar los parámetros de las funciones que mejor ajustan un conjunto de datos. Además la hoja de cálculo le permite seleccionar las opciones de presentar la ecuación en el gráfico y el valor de $\mathrm{R}$ cuadrado en el mismo (El factor $\mathrm{R}^{2}$ en cualquier modelo de regresión lineal indica qué tanta relación hay entre las variables, es decir, qué tanto se ve afectado el resultado $\mathrm{Y}$ al modificar $\mathrm{X}$, por consiguiente, si el valor de $\mathrm{R}^{2}$ es bajo, el modelo no es confiable porque no existe una fuerte relación entre $\mathrm{X}$ y $\mathrm{Y}$. El valor de $\mathrm{R}^{2}$ está siempre en el intervalo entre 0 a 1 , donde 0 indica que no existe ninguna relación entre X y Y, y 1 es la máxima 
relación), por tanto se debe buscar ajustar la curva a la función que permita que $\mathrm{R}^{2}$ tenga el valor más cercano a 1 .

1. Represente gráficamente en Excel el ancho de la hoja como función de la longitud, usando los datos de la tabla. ¿Es posible descubrir una relación lineal entre las dos variables? Marque las opciones de presentar la ecuación en el gráfico, así como el valor de $\mathrm{R}^{2}$ (verifique que este es muy cercano a 1). Escriba la relación obtenida de la forma $\mathrm{a}=\mathrm{kL}$, donde $\mathrm{k}$ es la constante de proporcionalidad entre las variables. ¿Qué significado físico tiene esta relación? (indague con sus compañeros para dar una respuesta consistente y bien fundamentada).

2. Represente gráficamente la masa de las hojas en función de su longitud. Como puede notar, la función obtenida esta vez no es una línea recta. Asigne el tipo de tendencia que mejor se ajuste a los datos (Lineal, Logarítmica, Polinomial, Potencia exponencial, etc.). Marque las opciones de presentar la ecuación en el gráfico, así como el valor de $\mathrm{R}^{2}$ (verifique que este es muy cercano a 1). Escriba la relación matemática obtenida de la forma $\mathrm{M}=\mathrm{kL}^{\mathrm{n}}$, debe expresar esta función con los valores de $\mathrm{k}$ y $\mathrm{n}$ obtenidos. Interprete físicamente la relación obtenida (escriba de forma argumentada).

3. Por último, agregue una nueva columna a la derecha de su tabla. Halle el área de la hoja suponiendo que puede aproximarse a la forma de una elipse (Área de una elipse $A=r_{1} r_{2}$, donde $\mathrm{r}_{1}$ es la mitad de la longitud, y $\mathrm{r}_{2}$ es la mitad del ancho. Ahora represente gráficamente la masa de las hojas en función de su área. Como puede ver es una relación lineal. Marque las opciones de presentar la ecuación en el gráfico, así como el valor de $\mathrm{R}^{2}$ (verifique que este es muy cercano a 1). Escriba la relación matemática obtenida de la forma $M=k A$. Suponiendo despreciable el espesor de las hojas comparado con sus otras dimensiones, determine la densidad superficial de las hojas de laurel, a partir de la relación matemática obtenida en la gráfica. Recuerde que densidad de masa superficial es $\sigma=M / A$. Si la densidad teórica de las hojas de laurel es $0,0046 \mathrm{~g} / \mathrm{cm}^{2}$, halle el error porcentual obtenido en el experimento. 


\section{Conclusiones}

Es la parte final de su reporte, en ella resume en pocas oraciones los resultados que alcanzó en el experimento. Resuma todos los resultados, no incluya todos los datos.

Inicie esta sección con algunas palabras como estas, "Los resultados demuestran que...", por ejemplo.

Resuma los puntos principales y verifique que se hayan cumplido los objetivos de la práctica. Dedique una conclusión a hablar sobre los porcentajes de error obtenidos y argumente sobre las posibles fuentes de error para este experimento. 


\section{Bibliografía}

Libro Digital: Volumen 1. Sears - Semansky, et al. Física universitaria con física moderna. Vol. 1. Editorial Pearson Education. Edición 13. Año 2013.

Link: www.ebooks7-24.com.aure.unab.edu.co/onlinepdfjs/view.aspx

Serway . R., et al., Física para ciencias e ingeniería. Vol. I. Editorial Cengage Learning. Edición 9. Año 2014.

Ohanian H., et al. Física para ingeniería y ciencias. Vol.1 Editorial Mc. Graw- HillInteramericana. Año 2009. 


\section{Sesión 2- Medidas Fundamentales}

\section{Temas de consulta - Medidas fundamentales (Preparación previa a la práctica)}

- Notación científica. Ejemplos.

- Exactitud y precisión.

- Cifras significativas. Ejemplos.

- Error absoluto y error relativo.

- Magnitudes fundamentales y magnitudes derivadas.

- Volumen de los siguientes cuerpos geométricos: Esfera, paralelepípedo, cilindro circular recto, cilindro hueco o anillo, pirámide.

- Densidades de algunas sustancias sólidas comunes (incluir la densidad de la madera).

- El calibrador o Pie de Rey

\section{Competencias}

Al finalizar la práctica el estudiante estará en capacidad de:

- Analizar un problema al cual dará solución con base en el análisis de datos y los temas consultados.

- Describir las cantidades físicas analizadas en su unidad correspondiente para distintos sistemas de unidades.

- Comparar ideas y opiniones para la toma de decisiones y planes respecto a la temática estudiada junto con sus compañeros de grupo.

\section{Materiales}

- Objetos sólidos de distintas formas geométricas.

- Calibrador. 
- Balanza.

- Flexómetro.

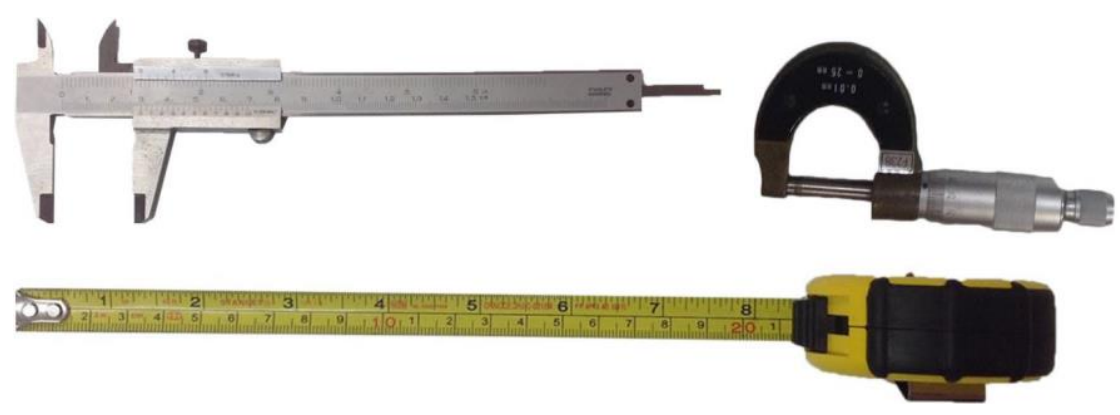

Figura 1. Instrumentos de medición: Calibrador (Pie de Rey), Flexómetro y Micrómetro.

\section{Procedimiento}

1. Identifique plenamente los instrumentos de medición mostrados en la Figura 1 a medida que el profesor instruye su uso, cerciórese de su funcionamiento y forma de medir los distintos objetos.

2. Utilice el calibrador para medir anchura, altura y grosor de cada una de las figuras geométricas suministradas registrando sus datos en la tabla 1.

3. Tome tres veces la medición de cada dimensión teniendo en cuenta la exactitud y precisión en cada medida.

4. Calcule el valor promedio de cada dimensión.

5. La balanza le permitirá conocer la masa de cada objeto. 
NOTA: Tenga en cuenta los valores máximos de medida que soporta la balanza, cerciórese de su funcionamiento y unidad de medida, consulte con su profesor o auxiliar.

6. Realice la medida del peso de cada trozo de madera procurando ser muy exacto.

7. Tabule los datos para la masa de cada objeto expresada en unidades del Sistema Internacional. Obtenga el valor promedio de la masa utilizada.

Tabla 1. Tablas de datos para las medidas fundamentales de cada uno de los objetos

PARALELEPÍPEDO

\begin{tabular}{|l|c|c|c|c|c|c|}
\hline \multirow{5}{*}{} & $\mathrm{a}(\mathrm{mm})$ & $\mathrm{b}(\mathrm{mm})$ & $\mathrm{c}(\mathrm{mm})$ & Masa $(\mathrm{kg})$ & $\begin{array}{c}\text { Densidad } \\
\left(\mathrm{Kg} / \mathrm{m}^{3}\right)\end{array}$ & $\begin{array}{c}\text { \% Error } \\
\text { Densidad }\end{array}$ \\
\cline { 2 - 6 } & & & & & \\
& & & & & & \\
\cline { 2 - 4 } & & & & & & \\
\hline Prom $(\mathrm{mm})$ & & & & & & \\
\hline
\end{tabular}

Volumen $=a * b * c=$

\section{ESFERA}

\begin{tabular}{|l|l|l|l|l|l|}
\hline & $\begin{array}{c}\text { D diámetro } \\
(\mathrm{mm})\end{array}$ & Radio $(\mathrm{m})$ & Masa $(\mathrm{kg})$ & $\begin{array}{c}\text { Densidad } \\
\left(\mathrm{Kg} / \mathrm{m}^{3}\right)\end{array}$ & $\begin{array}{c}\text { \% Error } \\
\text { Densidad }\end{array}$ \\
\cline { 2 - 5 } & & & & & \\
\cline { 2 - 2 } & & & & & \\
\hline Prom $(\mathrm{mm})$ & & & & \\
\hline Prom $(\mathrm{m})$ & & & & \\
Volumen $=\frac{4}{3} \pi r^{3}$
\end{tabular}


CILINDRO MACIZO

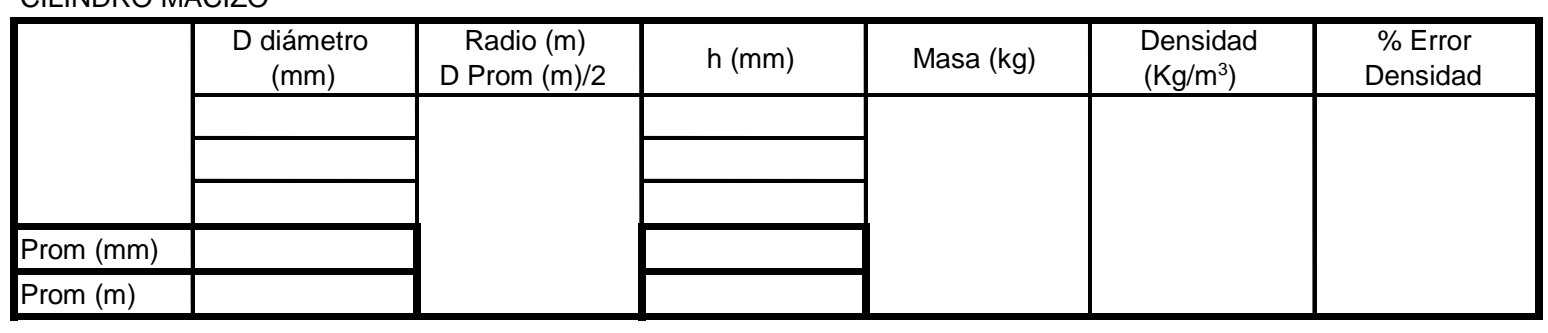

Volumen $=\pi r^{2} * h=$

CILINDRO HUECO

\begin{tabular}{|c|c|c|c|c|c|c|c|c|}
\hline \multirow[t]{2}{*}{ 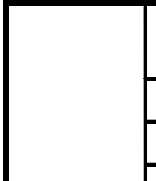 } & $\begin{array}{l}\text { D1 diámetro } \\
\text { Externo }(\mathrm{mm})\end{array}$ & $\begin{array}{l}\text { Radio } 1 \\
\text { Externo }(\mathrm{m})\end{array}$ & $\begin{array}{l}\text { D2 diámetro } \\
\text { Interno }(\mathrm{mm})\end{array}$ & $\begin{array}{c}\text { radio } 2 \\
\text { Interno }(\mathrm{m})\end{array}$ & $\mathrm{h}(\mathrm{mm})$ & Masa $(\mathrm{kg})$ & $\begin{array}{c}\text { Densidad } \\
\left(\mathrm{Kg} / \mathrm{m}^{3}\right)\end{array}$ & $\begin{array}{l}\% \text { Error } \\
\text { Densidad }\end{array}$ \\
\hline & & \multirow[t]{3}{*}{$R$} & & \multirow[t]{3}{*}{$r$} & & & & \\
\hline Prom (mm & & & & & & & & \\
\hline Prom (m) & & & & & & & & \\
\hline
\end{tabular}

Volumen $=\pi *\left(R^{2}-r^{2}\right) * h=$

PIRAMIDE

\begin{tabular}{|l|c|c|c|c|c|c|}
\hline \multirow{3}{*}{} & $\mathrm{a}(\mathrm{mm})$ & $\mathrm{b}(\mathrm{mm})$ & $\mathrm{h}(\mathrm{mm})$ & Masa $(\mathrm{kg})$ & $\begin{array}{c}\text { Densidad } \\
\left(\mathrm{Kg} / \mathrm{m}^{3}\right)\end{array}$ & $\begin{array}{c}\text { \% Error } \\
\text { Densidad }\end{array}$ \\
\cline { 2 - 5 } & & & & & \\
\cline { 2 - 4 } & & & & & \\
\hline
\end{tabular}

Volumen $=\frac{1}{3} * a * b * h=$

\section{Análisis}

1. Con los datos tabulados construya una gráfica de Masa Vs. Volumen para los distintos trozos de material utilizado.

2. Calcule la pendiente y exprese qué significado físico tiene. Registre esta información y compare 
los distintos tipos de madera.

3. ¿Qué proporcionalidad existe entre la masa y el volumen? ¿Se cumple para otros materiales?

4. ¿Qué porcentaje de error hubo en la densidad promedio de la madera?, compárela con la suministrada por el profesor.

5. Exprese sus observaciones y conclusiones sobre los aspectos físicos de su experimento teniendo en cuenta: la teoría, el procedimiento, la tabla de datos, las gráficas y las fuentes de error.

6. Plantee tres aplicaciones relacionadas con sus estudios de ingeniería que se relacionen con el fenómeno físico estudiado en esta sesión. 


\section{Bibliografía}

Libro Digital: Volumen 1. Sears - Semansky, et al. Física universitaria con física moderna. Vol. 1. Editorial Pearson Education. Edición 13. Año 2013.

Link: www.ebooks7-24.com.aure.unab.edu.co/onlinepdfjs/view.aspx

Serway. R., et al., Física para ciencias e ingeniería. Vol. I. Editorial Cengage Learning. Edición 9. Año 2014.

Ohanian H., et al. Física para ingeniería y ciencias. Vol.1 Editorial Mc. Graw- HillInteramericana. Año 2009. 


\section{Sesión 3- Vectores de fuerza}

Temas de consulta - Vectores de fuerza (Preparación previa a la práctica)

- Vectores

- Operaciones entre vectores

- Método del paralelogramo

- Método del polígono

- Método algebraico o analítico

- Concepto de fuerza

- Clases de fuerza

- Diagrama de fuerzas

\section{Competencias}

Al finalizar la práctica el estudiante estará en capacidad de:

- Aplicar las leyes de la adición de vectores usando diferentes estructuras para determinar fuerzas en equilibrio en situaciones reales utilizando diferentes métodos.

- Representar diagramas de fuerza para diferentes estructuras.

- Comparar ideas y opiniones para la toma de decisiones y planes respecto a la temática estudiada junto con sus compañeros de grupo.

\section{Materiales}

- Dinamómetros

- Soportes universales

- Diferentes masas 
- Regla métrica

- Lápiz

- Transportador

- Papel milimetrado

\section{Procedimiento}

1. Para el diseño indicado en la Figura $\mathbf{1}^{\boldsymbol{a}}$, mida con un transportador cada uno de los ángulos en la intersección de las cuerdas y realice un dibujo a escala para indicar dichos valores

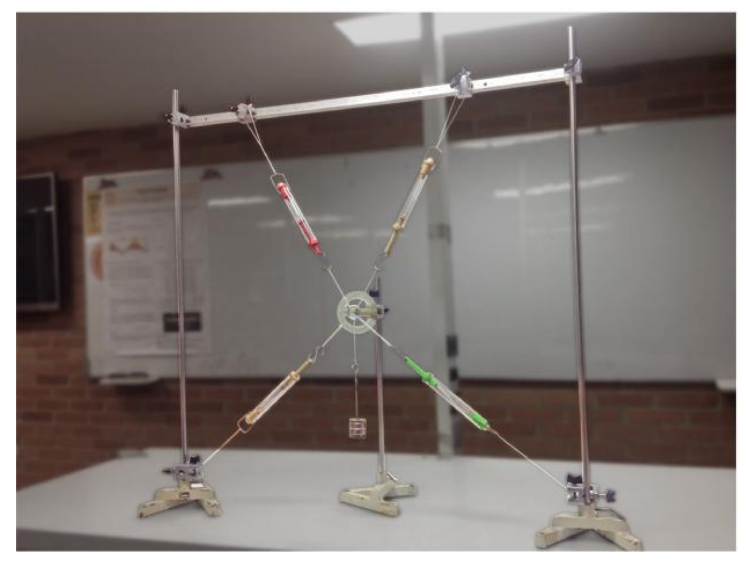

(a)

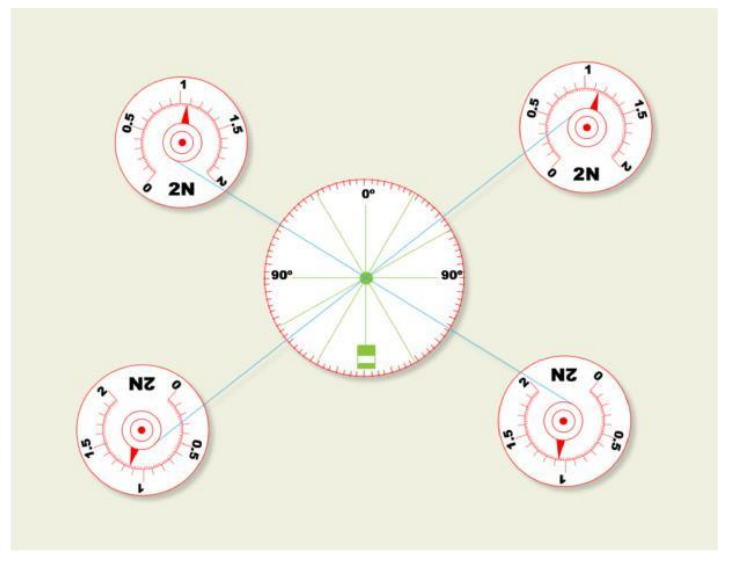

(b)

Imágenes de manuales electrónicos Laboratorio de Física UNAB

Figura 1. Esquema general para vectores de fuerza: (a) Dinamómetros alargados (b) Dinamómetros circulares

2. Utilice la tabla 1 para registrar los valores de las lecturas de cada dinamómetro en Dinas o en Newtons como lo muestra la figura 1a o 1b, y el peso en Newton de la masa suspendida, (Recuerde que el peso es el producto de la masa del objeto $(\mathrm{m})$ y la gravedad $\left(\mathrm{g}=9,81 \mathrm{~m} / \mathrm{s}^{2}\right)$ ).

3. Repita el proceso para otros dos montajes según las indicaciones del docente del Laboratorio y registre los valores en tablas similares. 
Tabla 1. Tabla de datos para los distintos montajes.

Montaje 1

VECTORES DE FUERZA

VECTORES

\begin{tabular}{|l|l|l|l|l|}
\hline & F Fuerza (N) & OAngulo(grados) & Fx (N) & Fy (N) \\
\hline F1 & & & & \\
\hline F2 & & & & \\
\hline F3 & & & & \\
\hline F4 & & & & \\
\hline F5 & & & & \\
\hline
\end{tabular}

\section{Análisis}

1. Empleando la dirección y magnitud de cada fuerza, construya un diagrama con las fuerzas que actúan dibujando líneas para representar la acción de las fuerzas con su correspondiente ángulo, utilizando un esquema similar al de la figura 1.

2. Use el método del paralelogramo para obtener la suma vectorial.

3. Use el método del Polígono para obtener la suma vectorial.

4. Use el método analítico para encontrar la fuerza resultante.

5. Compare el resultado anterior con cada uno de los métodos gráficos y evalúe la exactitud de la experiencia por medio del porcentaje de error para cada uno de los métodos estudiados.

6. Calcule el producto escalar y el producto vectorial para los vectores indicados en la tabla 2.

Tabla 2. Tabla de producto escalar y vectorial. 
PRODUCTO ENTRE VECTORES

\begin{tabular}{|l|l|l|}
\hline & $\overrightarrow{F a} \times \overrightarrow{F b}$ & $\overrightarrow{F a} \cdot \overrightarrow{F b}$ \\
\hline $\mathrm{F} 1, \mathrm{~F} 2$ & & \\
\hline $\mathrm{F} 3, \mathrm{~F} 4$ & & \\
\hline $\mathrm{F} 1, \mathrm{~F} 5$ & & \\
\hline
\end{tabular}

7. Exprese sus observaciones y conclusiones sobre los aspectos físicos de su experimento teniendo en cuenta: la teoría, el procedimiento, la tabla de datos, las gráficas y las fuentes de error.

8. Plantee tres aplicaciones relacionadas con sus estudios de ingeniería que se relacionen con el fenómeno físico estudiado en esta sesión.

\section{Bibliografía}

Libro Digital: Volumen 1. Sears - Semansky, et al. Física universitaria con física moderna. Vol. 1. Editorial Pearson Education. Edición 13. Año 2013.

Link: www.ebooks7-24.com.aure.unab.edu.co/onlinepdfjs/view.aspx 
Serway. R., et al., Física para ciencias e ingeniería. Vol. I. Editorial Cengage Learning. Edición 9. Año 2014.

Ohanian H., et al. Física para ingeniería y ciencias. Vol.1 Editorial Mc. Graw- HillInteramericana. Año 2009. 


\section{Sesión 4- Cinemática Unidimensional}

Temas a consultar - Movimiento unidimensional (Preparación previa a la práctica)

- Velocidad media, Velocidad instantánea

- Aceleración, aceleración media y aceleración instantánea

- Movimiento uniforme

- Movimiento uniformemente acelerado

\section{Competencias}

Al finalizar la práctica el estudiante estará en capacidad de:

- Caracterizar situaciones reales de movimientos en una dimensión (caso particular en el eje X)

- Describir en lenguaje científico la descripción de las diferentes clases de movimiento en una dimensión.

- Comparar ideas y opiniones para la toma de decisiones y planes respecto a la temática estudiada junto con sus compañeros de grupo.

\section{Materiales}

Carril de aire con sus accesorios (Bomba de aire, carros deslizadores)

Barreras de luz

Cronómetros

Flexómetro

\section{Procedimiento}

\section{A. Movimiento uniforme}

1. Según la figura 1, realice el montaje con los elementos suministrados, después de escuchar las 
instrucciones del profesor acerca del funcionamiento del equipo a utilizar.

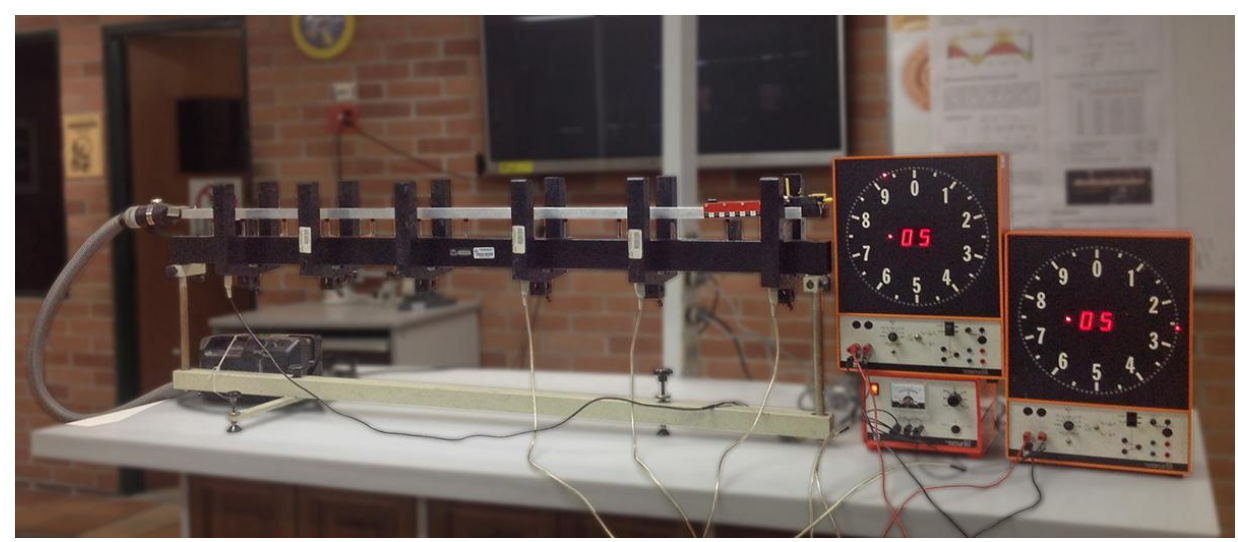

Figura 1. Esquema general para la cinemática en una dimensión.

2. Coloque sobre la mitad de la longitud del carril un nivel y manipule los tornillos de nivelación hasta que esté nivelado. Mida la longitud total del carril y divídala en no menos de 6 segmentos simétricos.

3. Coloque el objeto junto al dispositivo de choque ubicado al comienzo del carril. Use un indicador para las posiciones señalada en cada intervalo de distancia.

4. Suelte la palanca de impulso. Tabule los datos de espacio S y tiempo t. Repita el registro para el mismo desplazamiento una vez más.

5. Ejecute registros para los demás intervalos de espacio llenando la tabla 1, manteniendo la posición del dispositivo de choque constante. 
Tabla 1. Medidas para el movimiento uniforme

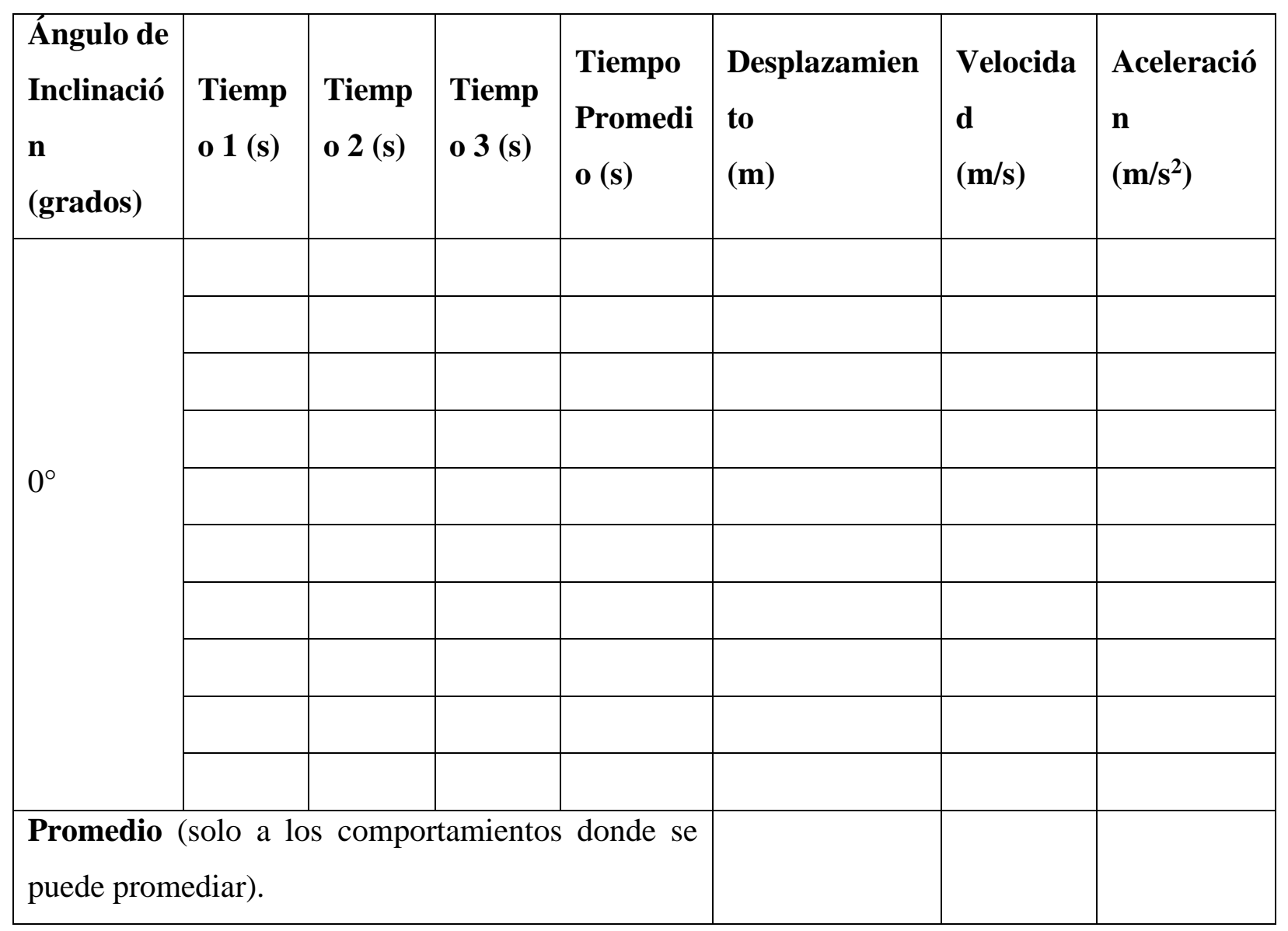

\section{B. Movimiento uniformemente acelerado}

\section{B.1 Movimiento uniformemente acelerado con $v 0 \neq 0$ y xo $=0$}

1. Eleve suavemente el carril colocando un taco al comienzo de él. Proceda según los numerales 3 a 5 del literal A. Tabule los datos de espacio (Desplazamiento) y tiempo.

$\mathrm{Vo} \neq \mathbf{0}$ y $\mathbf{X o}=\mathbf{0}$

\section{B.2 Movimiento uniformemente acelerado con vo= 0 y $\mathbf{x o}=0$}

1. No impulse el aerodeslizador con el dispositivo de choque, deje que el objeto ruede y proceda como en el literal A (sin impulso). 
2. Calcule velocidad y aceleración relacionando los datos experimentales de espacio $\mathrm{S}$ y el tiempo $\mathrm{t}$ promedio. Tabule los datos de espacio (Desplazamiento) y tiempo.

$\mathrm{Vo}=0$ y $\mathrm{Xo}=0$

\section{Análisis}

\section{A. Movimiento uniforme.}

1. Realice con sus datos experimentales las gráficas con los ejes comunes en la esquina inferior izquierda:
a) Espacio- Tiempo
b) Velocidad - Tiempo
c) Aceleración - Tiempo

2. Analice, justifique y obtenga la ecuación correspondiente para cada gráfica.

\section{B. Movimiento uniformemente acelerado.}

1. Construya con sus datos experimentales las gráficas correspondientes con los ejes comunes en cada uno de los siguientes casos:

a) Espacio - Tiempo (para los datos del numeral B1 y B2 )

b) Velocidad - Tiempo (para los datos del numeral B1 y B2 )

c) Aceleración - Tiempo (para los datos del numeral B1 y B2 )

2. Obtenga la ecuación correspondiente para cada gráfica. Analice y justifique su resultado. 
3. Exprese sus observaciones y conclusiones sobre los aspectos físicos de su experimento teniendo en cuenta: la teoría, el procedimiento, la tabla de datos, las gráficas y las fuentes de error.

4. Plantee tres aplicaciones relacionadas con sus estudios de ingeniería que se relacionen con el fenómeno físico estudiado en esta sesión.

\section{Bibliografía}

Libro Digital: Volumen 1. Sears - Semansky, et al. Física universitaria con física moderna. Vol. 1. Editorial Pearson Education. Edición 13. Año 2013.

Link: www.ebooks7-24.com.aure.unab.edu.co/onlinepdfjs/view.aspx 
Serway. R., et al., Física para ciencias e ingeniería. Vol. I. Editorial Cengage Learning. Edición 9. Año 2014.

Ohanian H., et al. Física para ingeniería y ciencias. Vol.1 Editorial Mc. Graw- HillInteramericana. Año 2009. 


\section{Sesión 5- Movimiento Semiparabólico}

Temas a consultar - Movimiento Semiparabólico (Preparación previa a la práctica)

- Movimiento en dos dimensiones

- Relación entre la cinemática del movimiento unidimensional y la cinemática bidimensional

- Representación geométricamente del movimiento con aceleración constante tiro parabólico.

\section{Competencias}

Al finalizar la práctica el estudiante estará en capacidad de:

- Integrar los conocimientos de análisis de movimiento en los ejes X, Y para analizar situaciones del mundo real.

- Describir en lenguaje científico la descripción de las diferentes clases de movimiento que conforman el movimiento en dos dimensiones.

- Comparar ideas y opiniones para la toma de decisiones y planes respecto a la temática estudiada junto con sus compañeros de grupo.

\section{Materiales}

Rampa en aluminio

Prensa

Soportes en V

Varillas de $1 \mathrm{~m}$ y de $20 \mathrm{~cm}$

Mordazas

Tabla

Papel carbón

Esferas de diferente material y peso 
Flexómetro

\section{Procedimiento}

\section{A. Cálculo del alcance máximo}

Realice el montaje experimental mostrado en la figura $\mathbf{1 b}$ con los elementos suministrados, después de escuchar las instrucciones del profesor acerca del experimento.

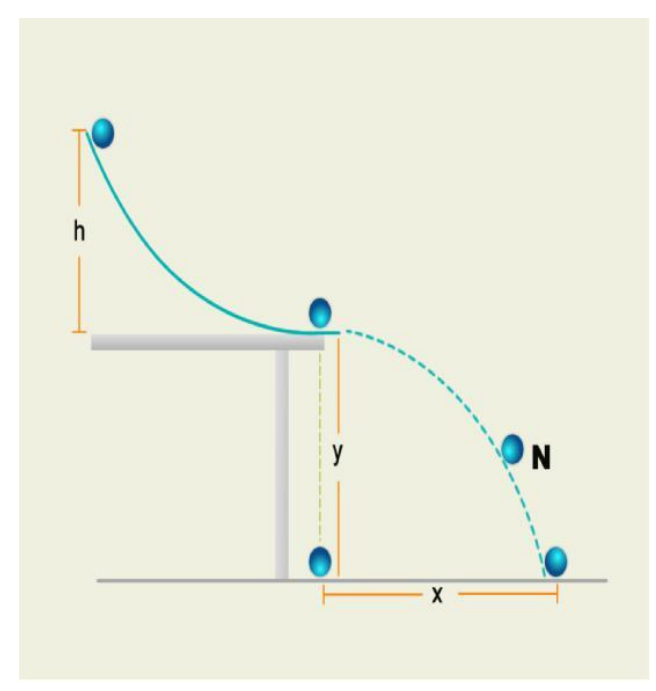

(a)

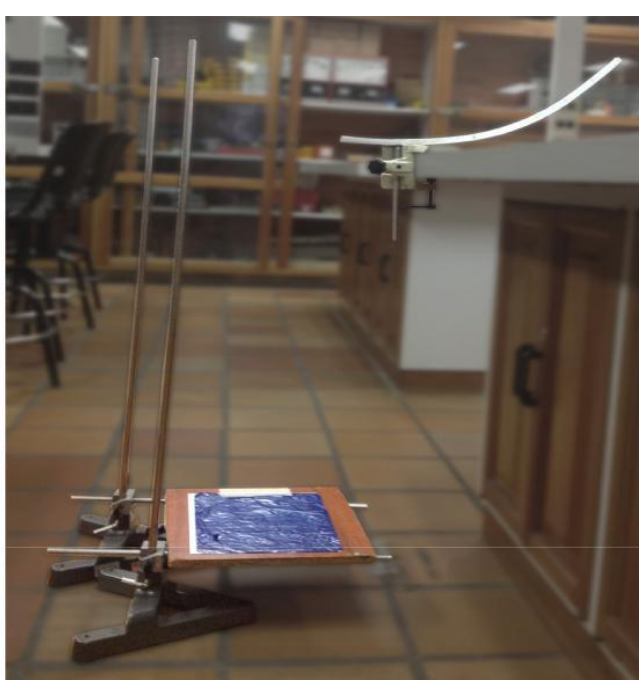

(b)

Figura 1. (a) Esquema general para el movimiento semiparabólico y las variables a medir. (b) Esquema experimental.

- Seleccione seis diferentes alturas desde la rampa disponible hasta el mesón según la figura 1a. Registre el alcance máximo. Repita la ejecución tres veces y registre valores $\left(\mathrm{X}_{1}, \mathrm{X}_{2}, \mathrm{X}_{3}\right)$ en la tabla 1.

Tabla 1. Medidas para el movimiento semiparabólico con diferentes alturas Y 
Movimiento semiparabólico con $\quad \mathbf{y}=\quad \mathbf{h}=$ variable

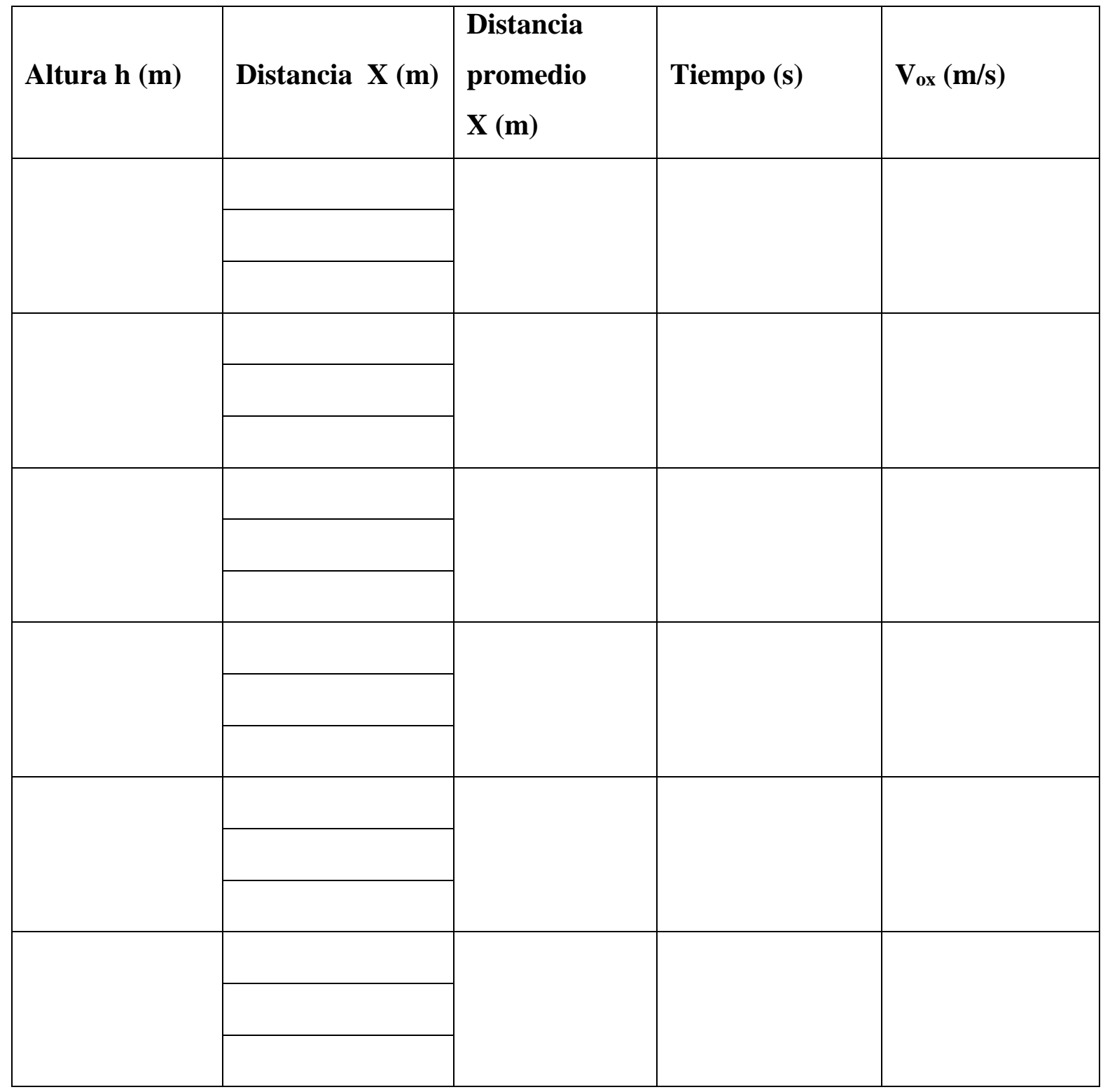

\section{B. Verificación experimental de las características en velocidad}

- Mida la altura del mesón hasta la base donde cae el objeto en ocho partes, mueva la base a cada una de estas posiciones y registre datos en la tabla 2 para dos lanzamientos. (Mantenga la altura 
ho máxima de la rampa constante).

Tabla 2. Medidas para el movimiento semiparabólico con Y constante

Movimiento semiparabólico $\operatorname{con} \mathbf{h}=$

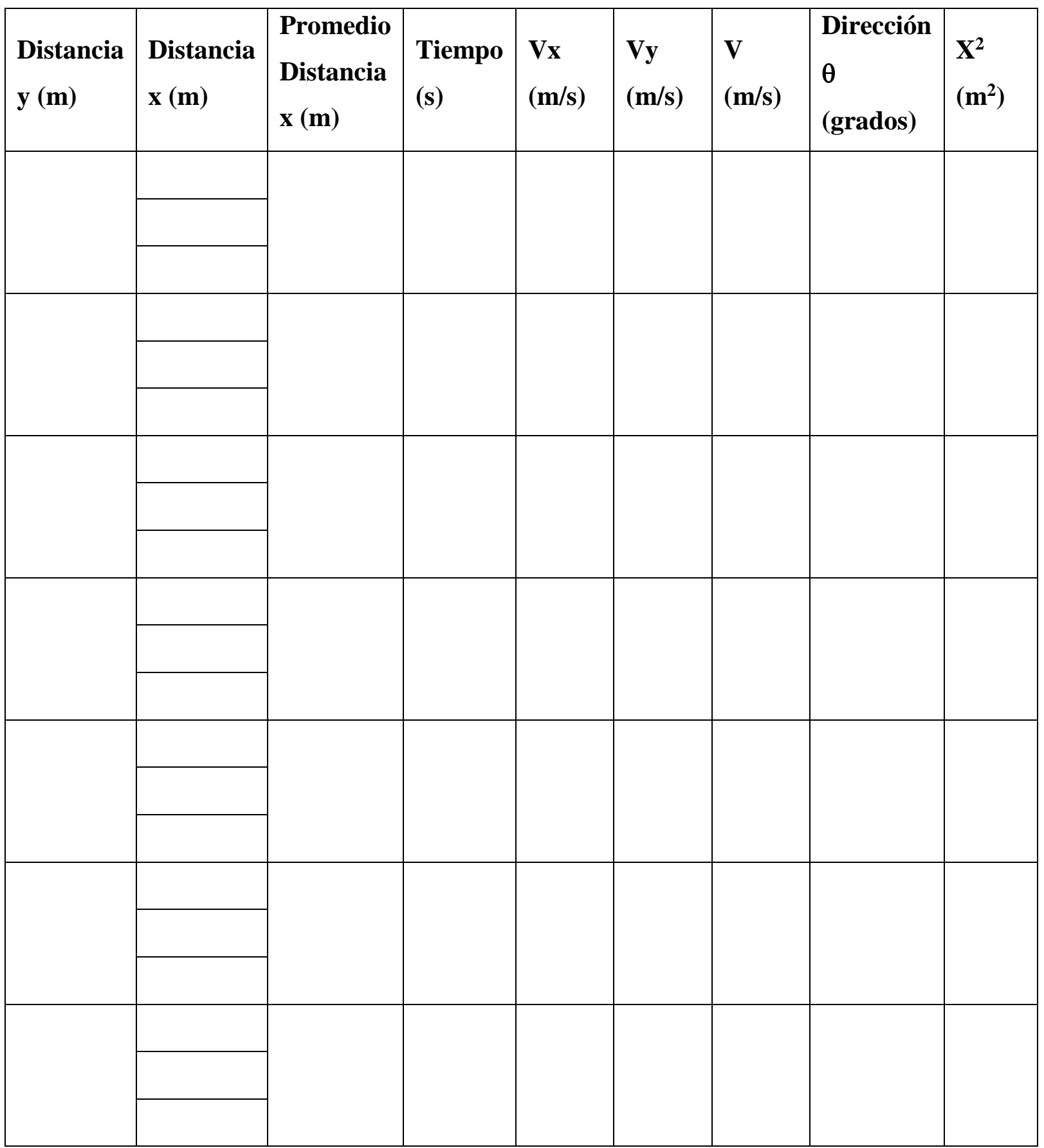




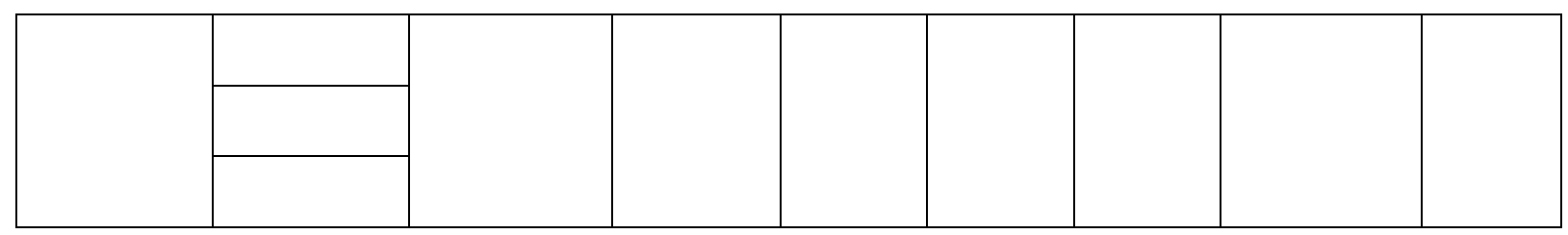

\section{Análisis}

Con sus datos experimentales y con la ayuda de las siguientes ecuaciones:

Ecuaciones:

\begin{tabular}{|c|c|}
\hline En $x$ & En $y$ \\
\hline$v_{\text {ox }}$ es constante & $a_{y}=g$ \\
$a_{x}$ es nula & $y=y_{0}+v_{o y} t+\frac{1}{2} g t^{2}$ \\
$x=x_{0}+v_{0 x} t$ & $v_{y}=v_{o y}+g t$ \\
& $v_{y}^{2}=v_{0 y}^{2}+2 g\left(y-y_{0}\right)$ \\
\hline
\end{tabular}

1. Calcule los parámetros necesarios para completar las casillas indicadas en las tablas 1 y 2. Tome el valor de la gravedad como $9,81 \mathrm{~m} / \mathrm{s}^{2}$.

2. Grafique y Vs. $\mathbf{t}^{2} ; V_{\mathbf{y}}$ Vs. t; x Vs. t; y Vs. $\mathbf{x}^{2}$

3. Realice el análisis correspondiente a cada gráfica y justifíquelo matemáticamente con la ecuación obtenida.

4. Halle los porcentajes de error a las variables de las gráficas lineales. Analice y discuta sus resultados.

5. De acuerdo a la teoría, los objetivos, el procedimiento, los datos obtenidos y calculados y sus gráficas, observe, analice y concluya sin perder de vista los aspectos físicos de este experimento. 
6. Plantee tres aplicaciones relacionadas con sus estudios de ingeniería que se relacionen con el fenómeno físico estudiado en esta sesión. 


\section{Bibliografía}

Libro Digital: Volumen 1. Sears - Semansky, et al. Física universitaria con física moderna. Vol. 1. Editorial Pearson Education. Edición 13. Año 2013.

Link: www.ebooks7-24.com.aure.unab.edu.co/onlinepdfjs/view.aspx

Serway. R., et al., Física para ciencias e ingeniería. Vol. I. Editorial Cengage Learning. Edición 9. Año 2014.

Ohanian H., et al. Física para ingeniería y ciencias. Vol.1 Editorial Mc. Graw- HillInteramericana. Año 2009. 


\section{Sesión 6- Conservación de energía}

Temas De Consulta - Conservación de energía (Preparación previa a la práctica)

- Ecuaciones de la energía

- Análisis físico de la energía cinética y potencial

- Ley de conservación de la energía

- Energía mecánica

- Ecuaciones del movimiento de proyectiles

\section{Competencias}

- Hallar experimentalmente la relación entre la variación de energía potencial gravitatoria y la variación de la energía cinética de un cuerpo que cae libremente.

- Describir en lenguaje científico el principio de conservación de energía para otros modelos.

- Comparar ideas y opiniones para la toma de decisiones y planes respecto a la temática estudiada junto con sus compañeros de grupo.

\section{Materiales}

- Rampa de aluminio

- Prensa

- Esfera

- Flexómetro

- Papel carbón y blanco

\section{Procedimiento}

1. Realice el montaje mostrado en la figura 1a, seleccione seis diferentes alturas desde la rampa hasta el mesón y marque con un lápiz sobre la rampa metálica mostrada en la figura $\mathbf{1 b .}$ 


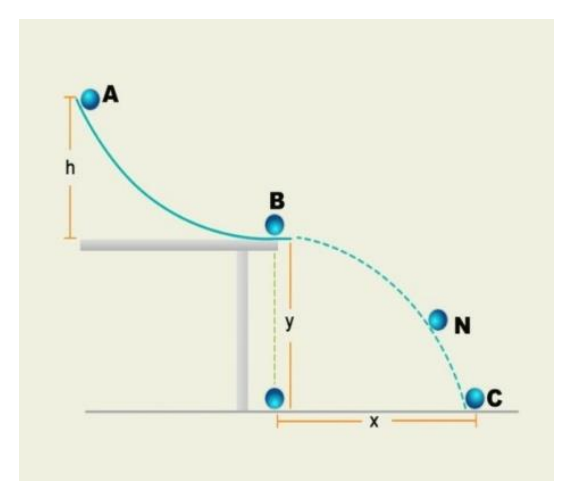

(a)

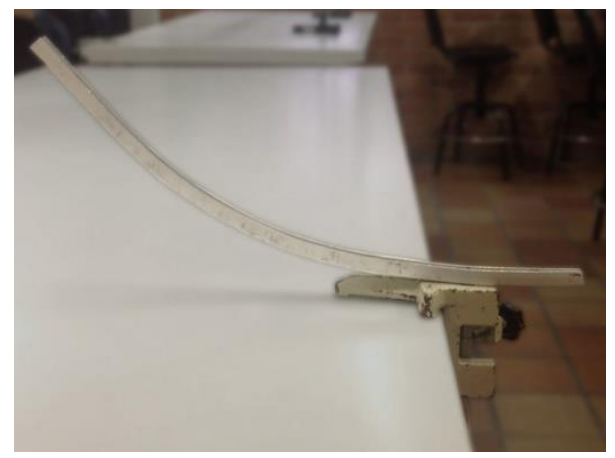

(b)

Figura 1. (a) Esquema general para el movimiento semiparabólico y las variables a medir. (b) Esquema experimental.

2. Inicie con la mayor altura sobre la rampa del montaje mostrado en la figura $\mathbf{1}$ y suelte desde allí la esfera. Registre el alcance máximo midiendo desde el nivel del suelo hasta donde golpea el papel carbón. Repita la ejecución tres veces y registre valores $\left(\mathrm{X}_{1}, \mathrm{X}_{2}, \mathrm{X}_{3}\right)$ en la tabla $\mathbf{1}$.

Repita el proceso anterior para la seis diferentes alturas de la rampa hasta el mesón.

3. Tome la altura Y, desde el punto final de la rampa hasta el suelo.

4. Mida la masa de la esfera.

5. Con las ecuaciones del movimiento de proyectiles calcule el tiempo de vuelo de la partícula.

6. Calcule la velocidad inicial (horizontal) de la esfera para cada altura $\mathbf{h}$, por medio del alcance horizontal medido y el tiempo hallado en el punto anterior y registre estos valores también en la tabla 2. Nota: De aquí en adelante se usará la tabla 2.

7. Calcule la Energía mecánica A con respecto al punto de referencia indicado por el profesor.

8. Calcule la Energía mecánica $\mathbf{B}$ con respecto al punto de referencia indicado por el profesor.

9. Calcule la Energía mecánica $\mathbf{C}$ con respecto al punto de referencia indicado por el profesor.

10. Halle la velocidad inicial (horizontal) de la esfera para cada altura $\mathbf{h}$, utilizando el principio de conservación de la energía mecánica.

11. Halle el error relativo para cada una de las velocidades iniciales encontradas, tomando como valor teórico el encontrado por medio del principio de conservación de la energía y como valor experimental el encontrado por medio de las ecuaciones cinemáticas del movimiento de proyectiles. 
Tabla 1. Medidas para el movimiento semiparabólico

Movimiento Semiparabólico $\mathbf{y}=\quad \mathbf{h}=$ variable

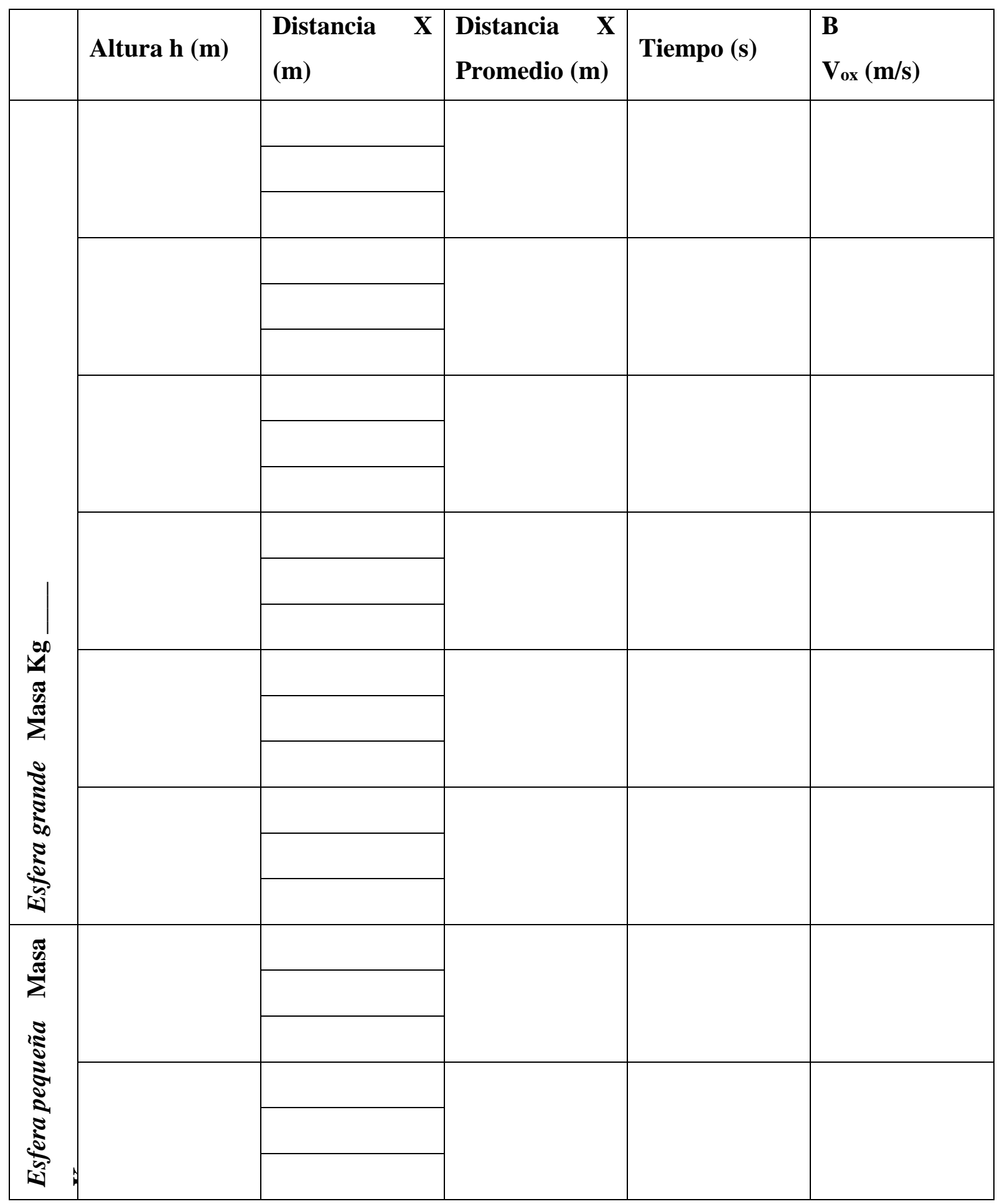




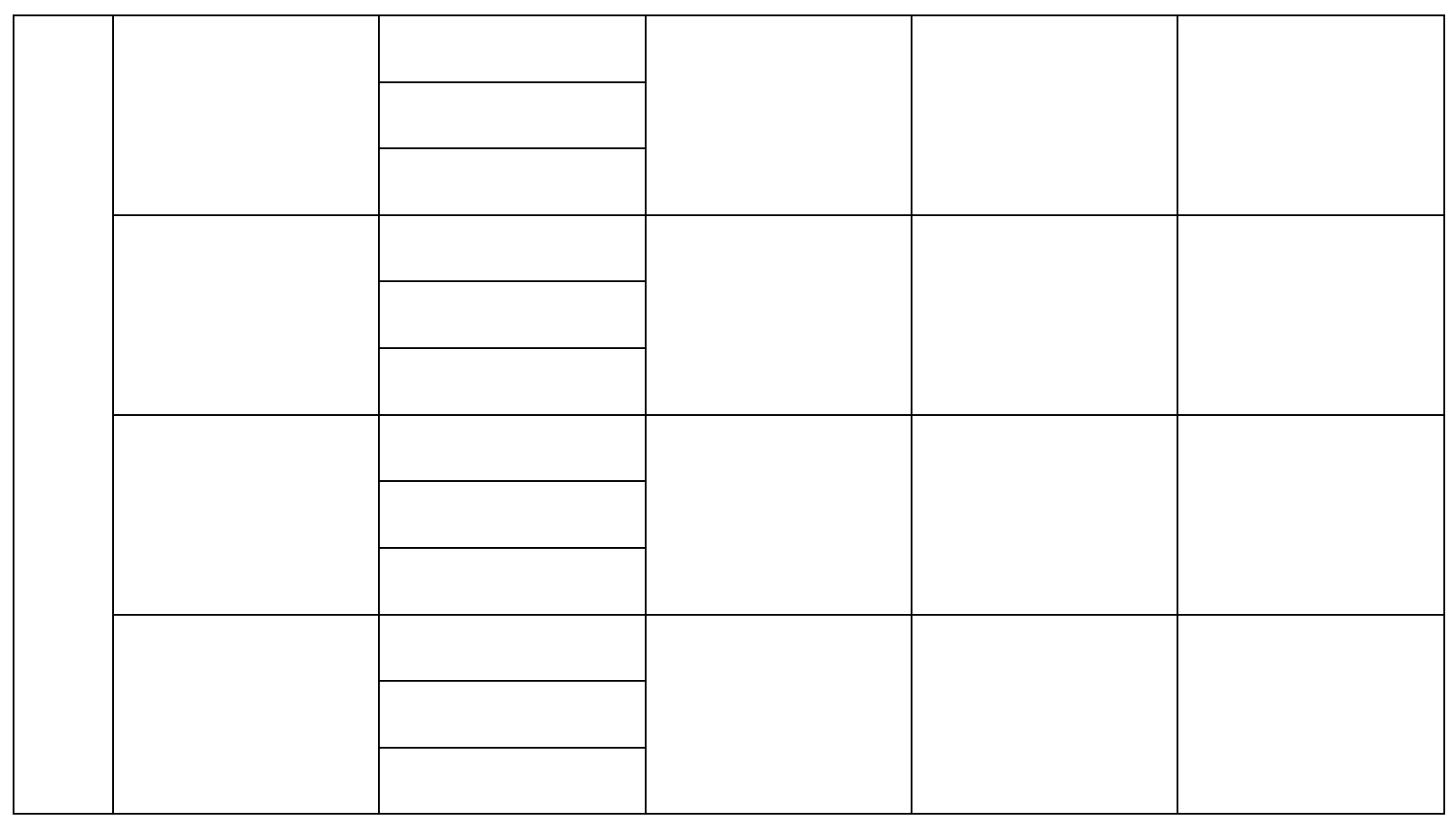

Tabla 2. Datos y cálculos para el análisis de energía

Movimiento Conservación de la energía $\quad \mathbf{V}_{\mathbf{A}}=\mathbf{0}$

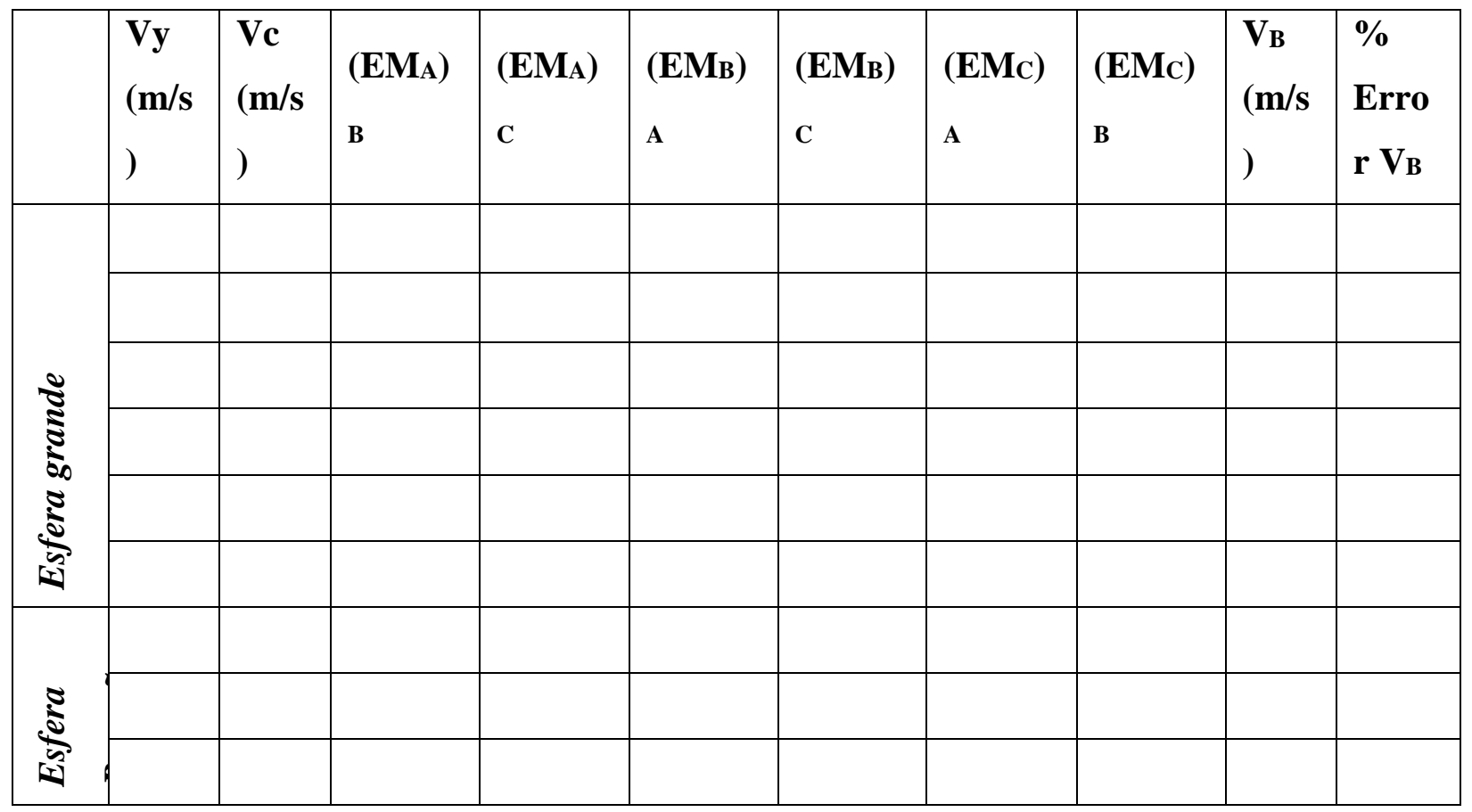




\begin{tabular}{|l|l|l|l|l|l|l|l|l|l|l|}
\hline & & & & & & & & & \\
\hline & & & & & & & & & \\
\hline & & & & & & & & & \\
\hline
\end{tabular}

Recuerde las Ecuaciones cinemáticas generales (de acuerdo al sistema de referencia de la gráfica)

\begin{tabular}{|c|c|}
\hline Enx & En $y$ \\
& \\
\hline$v_{\text {ox }}$ es constante & $a_{y}=g$ \\
$a_{x}$ es nula & $y=y_{0}+v_{o y} t+\frac{1}{2} g t^{2}$ \\
$x=x_{0}+v_{0 x} t$ & $v_{y}=v_{o y}+g t$ \\
& $v_{y}^{2}=v_{0 y}^{2}+2 g\left(y-y_{0}\right)$ \\
\hline
\end{tabular}

Tenga en cuenta las ecuaciones para la Energía:

\begin{tabular}{|l|c|}
\hline Energía Cinética & $E_{c}=\frac{1}{2} m v^{2}$ \\
\hline Energía Potencial Gravitacional & $E_{p}=m g h$ \\
\hline Energía Mecánica & $E_{M}=E_{c}+E_{p}$ \\
\hline
\end{tabular}

NOTA: Identifique el nivel de referencia escogido para el cálculo de la Energía en cada punto del sistema.

\section{Análisis}

1. Realice la gráfica $\mathrm{EM}_{\mathrm{B}}$ vs. $\mathrm{EM}_{\mathrm{A}}, \mathrm{EM}_{\mathrm{B}}$ vs. $\mathrm{EM}_{\mathrm{C}}$.

2. Analice y describa el comportamiento de la gráfica.

3. Elabore un comentario acerca de los resultados obtenidos y enumere posibles fuentes de error 
en la experiencia.

4. Exprese sus observaciones y conclusiones sobre los aspectos físicos de su experimento teniendo en cuenta: la teoría, el procedimiento, la tabla de datos, las gráficas y las fuentes de error.

5. Plantee tres aplicaciones relacionadas con sus estudios de ingeniería que se relacionen con el fenómeno físico estudiado en esta sesión. 


\section{Bibliografía}

Libro Digital: Volumen 1. Sears - Semansky, et al. Física universitaria con física moderna. Vol. 1. Editorial Pearson Education. Edición 13. Año 2013.

Link: www.ebooks7-24.com.aure.unab.edu.co/onlinepdfjs/view.aspx

Serway. R., et al., Física para ciencias e ingeniería. Vol. I. Editorial Cengage Learning. Edición 9. Año 2014.

Ohanian H., et al. Física para ingeniería y ciencias. Vol.1 Editorial Mc. Graw- HillInteramericana. Año 2009. 


\section{Sesión 7- Equilibrio estático}

Temas de consulta - Equilibrio estático (Preparación previa a la práctica)

- Fuerzas paralelas

- Momento de torsión

- Primera ley de Newton

- Condiciones para el equilibrio estático

\section{Competencias}

Al finalizar la práctica el estudiante estará en capacidad de:

- Resolver problemas donde se apliquen momentos de torsión para sistemas sencillos que se encuentran en equilibrio.

- Describir en lenguaje científico los principios del equilibrio estático para un sistema de fuerzas.

- Comparar ideas y opiniones para la toma de decisiones y planes respecto a la temática estudiada junto con sus compañeros de grupo.

\section{Materiales}

Dinamómetros de diferentes escalas

Mordazas

Varillas de $1 \mathrm{~m}$

Bases triangulares

Masas de diferentes valores

Soporte para las masas

Balanza

Flexómetro 


\section{Procedimiento}

1. Construya el montaje indicado por el profesor de acuerdo a la figura 1a o 1b según indicaciones del profesor, teniendo en cuenta que los dinamómetros pueden colgarse de la varilla metálica utilizando las mordazas suministradas. Asegúrese de que el mecanismo del dinamómetro pueda moverse libremente.
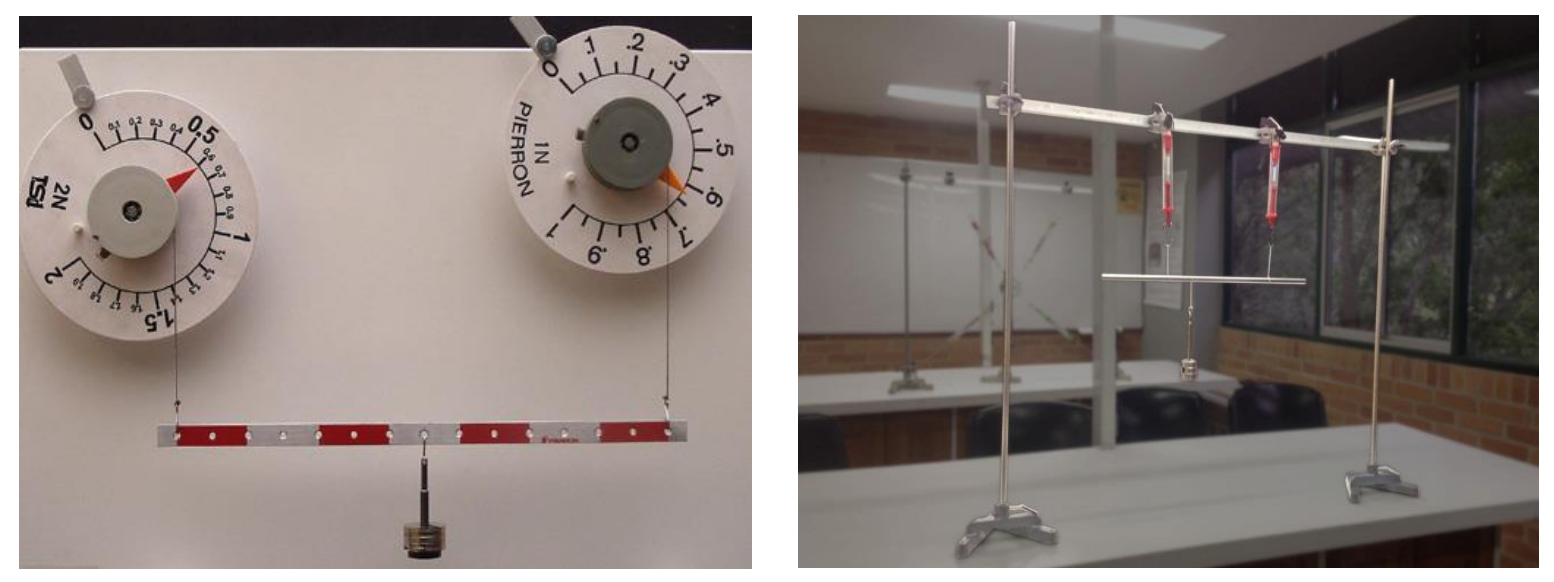

http://www.heurema.com/PDF/PDF9-

FParalelas/fotospdf10/690b2-2-7W.jpg

(a)

(b)

Figura 1. (a) Esquema experimental 1. (b) Esquema experimental 2.

2. Observe las lecturas de la fuerza en los dinamómetros. Registre estos valores en la tabla 1.

Tabla 1. Medidas para el análisis del equilibrio estático a partir de varios puntos de referencia.

\section{Desde A.}




\begin{tabular}{|l|l|l|l|l|l|}
\hline $\begin{array}{l}\text { Fuerzas } \\
(\mathrm{N})\end{array}$ & $\begin{array}{l}\mathrm{r} \text { Distancias } \\
(\mathrm{m})\end{array}$ & $\begin{array}{l}\theta \text { Ángulo } \\
(\text { grados })\end{array}$ & $\begin{array}{l}\tau \text { Torque } \\
\left(\mathrm{N}^{*} \mathrm{~m}\right)\end{array}$ & Gráficos \\
\hline $\mathrm{F}_{1}$ & & & & & \\
\hline $\mathrm{F}_{2}$ & & & & & \\
\hline $\mathrm{F}_{3}$ & & & & & \\
\hline $\mathrm{F}_{4}$ & & & & & \\
\hline $\mathrm{F}_{5}$ & & & & & \\
\hline $\mathrm{F}_{6}$ & & & & & \\
\hline$(\mathrm{W})$ & & & & & \\
\hline
\end{tabular}

3. Cuelgue una masa en el centro de la barra. Observe las lecturas de los dinamómetros en los diferentes puntos cuando el aparato está en equilibrio. Apunte estos valores en la tabla 1 como letras finales. No olvide registrar el valor de la fuerza de la masa suspendida y de la barra.

4. Mida y registre las distancias correspondientes respecto al punto de referencia indicado.

5. Recuerde que el momento de torsión es igual al producto de la lectura real del dinamómetro y la distancia desde el punto de apoyo escogido hasta el dinamómetro, teniendo en cuenta el signo según sea en la dirección de las manecillas del reloj (-) o en dirección contraria a las manecillas del reloj (+). Calcule los momentos de torsión en ambas direcciones y apunte los valores en la tabla.

6. Ahora tome dos masas de diferente magnitud y ubíquelas en dos posiciones diferentes de la barra hasta obtener el equilibrio, dejando los dinamómetros en los puntos iniciales. Registre las lecturas reales de los dinamómetros, al igual que el valor de las masas suspendidas. 
7. Si la barra queda inclinada debe encontrar el ángulo que hace con respecto a la horizontal. Mida la longitud total de la barra. Registre estos valores en su tabla de datos.

8. Repita los pasos del 2 al 7 para otros montajes indicados por el profesor.

\section{Análisis}

1. ¿Cómo el sistema en cada ensayo estaba en equilibrio? ¿Qué condiciones han sido cumplidas?

2. Utilice las dos condiciones de equilibrio estático para comprobar que cada uno de los sistemas empleados están en equilibrio.

3. Compare los valores absolutos de los momentos de torsión en un punto de referencia diferente al anterior.

4. ¿Qué relación debe existir entre los momentos de torsión en contra y en la misma dirección del movimiento de las manecillas del reloj si el sistema está en equilibrio?

5. ¿Cuándo los sistemas en sus ensayos estuvieron en equilibrio? ¿Cuánto trabajo se realizó? Explique su respuesta.

6. Exprese sus observaciones y conclusiones sobre los aspectos físicos de su experimento teniendo en cuenta: la teoría, el procedimiento, la tabla de datos, las gráficas y las fuentes de error.

7. Plantee tres aplicaciones relacionadas con sus estudios de ingeniería que se relacionen con el fenómeno físico estudiado en esta sesión. 


\section{Bibliografía}

Libro Digital: Volumen 1. Sears - Semansky, et al. Física universitaria con física moderna. Vol. 1. Editorial Pearson Education. Edición 13. Año 2013.

Link: www.ebooks7-24.com.aure.unab.edu.co/onlinepdfjs/view.aspx

Serway. R., et al., Física para ciencias e ingeniería. Vol. I. Editorial Cengage Learning. Edición 9. Año 2014.

Ohanian H., et al. Física para ingeniería y ciencias. Vol.1 Editorial Mc. Graw- HillInteramericana. Año 2009. 